ARTICLE

https://doi.org/10.1038/s41467-019-13931-7

\title{
DAF-16/FOXO requires Protein Phosphatase 4 to initiate transcription of stress resistance and longevity promoting genes
}

Ilke Sen 1,2,3, Xin Zhou (1) 1,2, Alexey Chernobrovkin ${ }^{4}$, Nataly Puerta-Cavanzo (i) ${ }^{3}$, Takaharu Kanno ${ }^{2,5}$, Jérôme Salignon ${ }^{1,2}$, Andrea Stoehr (10 1,2, Xin-Xuan Lin 1,2, Bora Baskaner ${ }^{1}$, Simone Brandenburg ${ }^{3}$, Camilla Björkegren (10) 2,5, Roman A. Zubarev ${ }^{4}$ \& Christian G. Riedel (1) 1,2,3*

In C. elegans, the conserved transcription factor DAF-16/FOXO is a powerful aging regulator, relaying dire conditions into expression of stress resistance and longevity promoting genes. For some of these functions, including low insulin/IGF signaling (IIS), DAF-16 depends on the protein SMK-1/SMEK, but how SMK-1 exerts this role has remained unknown. We show that SMK-1 functions as part of a specific Protein Phosphatase 4 complex (PP4SMK-1). Loss of PP4 ${ }^{\mathrm{SMK}-1}$ hinders transcriptional initiation at several DAF-16-activated genes, predominantly by impairing RNA polymerase II recruitment to their promoters. Search for the relevant substrate of PP4 ${ }^{\mathrm{SMK}-1}$ by phosphoproteomics identified the conserved transcriptional regulator SPT-5/SUPT5H, whose knockdown phenocopies the loss of PP4SMK-1. Phosphoregulation of SPT-5 is known to control transcriptional events such as elongation and termination. Here we also show that transcription initiating events are influenced by the phosphorylation status of SPT-5, particularly at DAF-16 target genes where transcriptional initiation appears rate limiting, rendering PP4 ${ }^{\mathrm{SMK}-1}$ crucial for many of DAF-16's physiological roles.

\footnotetext{
${ }^{1}$ Integrated Cardio Metabolic Centre (ICMC), Department of Medicine, Karolinska Institute, Blickagången 6, 14157 Huddinge, Sweden. ${ }^{2}$ Department of Biosciences and Nutrition, Karolinska Institute, Blickagången 16, 14157 Huddinge, Sweden. ${ }^{3}$ European Research Institute for the Biology of Ageing (ERIBA), University Medical Center Groningen (UMCG), University of Groningen, Antonius Deusinglaan, 1, 9713AV Groningen, The Netherlands. ${ }^{4}$ Department of Medical Biochemistry and Biophysics, Karolinska Institute, Solnavägen 9, 17165 Solna, Sweden. ${ }^{5}$ Department of Cellular and Molecular Biology, Karolinska Institute, Solnavägen 9, 17165 Solna, Sweden. *email: christian.riedel@ki.se
} 
nsulin/IGF-like signaling (IIS) is one of the most prominent aging-regulatory pathways. Conserved from worms to mammals ${ }^{1,2}$, it has critical roles in the regulation of metabolism, reproduction, stress resistance, and longevity ${ }^{3,4}$. In particular the stress resistance and longevity phenotypes are controlled through a conserved downstream transcription factor called DAF-16/FOXO. Under normal conditions, IIS is active and phosphorylates DAF-16 via AKT and SGK kinases, leading to the sequestration of DAF-16 in the cytoplasm through interaction with 14-3-3, away from its target genes. However, under dire conditions-those leading to low IIS in particular-phosphorylation is reduced, allowing DAF-16 to translocate into the nucleus. There it binds to the promoters and regulates transcription of its target genes, predominantly inducing genes that contribute to stress response mechanisms and promote longevity ${ }^{5,6}$.

Although nuclear translocation of DAF-16 is a prerequisite, it is not sufficient for DAF-16 to regulate its target genes ${ }^{7}$. Additional regulators and cofactors are needed. One of the most powerful positive regulators of DAF-16, the conserved protein SMK-1/SMEK, was described already a decade ago ${ }^{8}$. Under low IIS, SMK-1 is required for DAF-16-driven longevity and the resistance to oxidative stress, to ultraviolet (UV) irradiation, and to pathogens. Interestingly though, it is not required for DAF-16 to promote thermotolerance, to promote dauer formation, or to delay reproduction ${ }^{8}$. This suggests that SMK-1 genetically interacts with DAF-16 but regulates only parts of its functions, likely by influencing the expression of only a specific subset of its target genes.

Despite this prior work and the interesting phenotypes observed, the mechanism by which SMK-1 would influence gene expression has remained unknown. Here we show that SMK-1 acts as part of a specific Protein Phosphatase 4 complex, PP4 ${ }^{\text {SMK- }}$ 1 , which binds and dephosphorylates the transcriptional regulator SPT-5. Under low IIS, this dephosphorylation of SPT-5 promotes transcription initiating events, in particular RNA Pol II (Pol II) recruitment at several DAF-16 target genes, and thereby promotes a large part of DAF-16's functions.

\section{Results}

SMK-1 is part of a nuclear Protein Phosphatase 4 complex. To gain insight into the mechanism by which SMK-1 promotes DAF-16 functions, we sought to identify proteins bound to SMK-1. We grew Caenorhabditis elegans expressing SMK-1::mCherry to large quantity, lysed them, and conducted an immunoprecipitation (IP) using anti-mCherry antibody. The precipitate was then analyzed by silver staining (Fig. 1a) and tandem mass spectrometry (MS/MS, Fig. 1b). By this approach, we found 16 proteins that co-purified with SMK-1:: mCherry (Fig. 1b, c, see Supplementary Table 2 for an independent repeat). The most abundant of these were subunits of Protein Phosphatase 4 (PP4); namely the catalytic subunits $\mathrm{PPH}-4.1$ and $\mathrm{PPH}-4.2$, and the regulatory subunit PPFR-2 (Fig. 1b, c). According to previous work in yeast and mammals, PP4 complexes exist in different defined subunit compositions. Each complex contains a catalytic subunit of PP4, of which the closest C. elegans orthologs are $\mathrm{PPH}-4.1$ and $\mathrm{PPH}-4.2$, and it contains a selection of four regulatory subunits, which help to target PP4 to its substrates ${ }^{9-11}$. Closest C. elegans orthologs to three of these regulatory subunits are PPFR-1, PPFR-2, and PPFR-4. Strikingly, closest ortholog to the fourth regulatory subunit is SMK-1. Together with our IP-MS/MS data, this suggests that SMK-1 is actually a regulatory subunit of a specific PP4 complex in C. elegans, hereafter referred to as $\mathrm{PP} 4^{\mathrm{SMK}-1}$. This complex has a unique subunit composition, containing either of the catalytic subunits and the regulatory subunits SMK-1 and PPFR-2, but not PPFR-1 or PPFR-4 (see also Fig. 1c). Next, we sought to confirm this result by independent IP-MS/MS experiments, using a different epitope tag on SMK-1 and a different affinity matrix (SMK-1::GFP and GFP-Trap, respectively). Given that SMK-1 is required for DAF-16 functions when IIS activity is low but not when it is high $^{8}$, we further tested in this experiment whether the incorporation of SMK-1 into the PP4 ${ }^{\mathrm{SMK}-1}$ complex is IISdependent, by conducting the IPs from either daf-2(e1370) (a mutant of the insulin/IGF receptor, resulting in low IIS) or daf18(mg198) (a mutant of PTEN, resulting in high IIS) animals. We confirmed the existence and composition of the PP4 $4^{\mathrm{SMK}-1}$ complex and found that the formation of this complex was not affected by IIS activity (Supplementary Table 3). As a final demonstration of how readily SMK-1 incorporates into PP4 $4^{\text {SMK-1 }}$ complexes in vivo, we expressed a minimal C. elegans PP4 ${ }^{\text {SMK-1 }}$ complex comprised of SMK-1::HA 3 , PPFR-2, and PPH-4.1::TAP in Saccharomyces cerevisiae, purified PPH4.1-TAP by IgG pulldown, and then detected SMK-1::HA 3 by western blotting. SMK-1::HA $\mathrm{HA}_{3}$ specifically co-purified with PPH-4.1::TAP (Supplementary Fig. 9d).

A surprising observation in these SMK-1 IPs was the prominent presence of the phosphatase subunit PAA-1 (Fig. 1b, c, Supplementary Tables 2, 3). PAA-1 is a well-established scaffold subunit of Protein Phosphatase 2A (PP2A) complexes ${ }^{12}$ that had not been found in PP4 complexes before. Our data suggests that, at least in C. elegans, PAA-1 may also be part of PP4 complexes, or alternatively that SMK-1 may be part not only of PP4 $4^{\mathrm{SMK}-1}$ but also a PP2A-related complex. Future studies will have to evaluate this.

Next, we were interested in the localization of the PP4 4 SMK-1 complex, to get an indication of the tissues and cellular compartments, in which the complex regulates DAF-16 function under low IIS. We studied wild type and daf-2(e1370) animals expressing either SMK-1::GFP or PPH-4.1::GFP under their endogenous promoters. Animals expressing DAF-16::GFP served as additional control. We found that similar to DAF-16::GFP also SMK-1::GFP and PPH-4.1::GFP were globally expressed (Fig. 1d, Supplementary Fig. 1, see also $\left.{ }^{8,13}\right)$. Within cells, SMK-1::GFP localized strictly to the nucleus, while PPH-4.1::GFP could be seen in both the nucleus and cytoplasm (Fig. 1d, Supplementary Fig. 1). Expression pattern and subcellular localization of SMK-1:: GFP and PPH-4.1::GFP were not affected by changes in IIS activity (Fig. 1d, Supplementary Fig. 1). This data showed that under low IIS the PP4 ${ }^{\text {SMK-1 }}$ complex has the opportunity to form in all tissues, and the intracellular localization patterns of its subunits suggest that the complex resides and exerts its functions in the nucleus.

PP4 ${ }^{\text {SMK-1 }}$ is required for DAF-16 to promote longevity. In light of this complex formation, we tested whether SMK-1 contributes to DAF-16's functions as part of PP4 $4^{\mathrm{SMK}-1}$-in particular through the complex's catalytic activity. First, we explored the role of $\mathrm{PP} 4{ }^{\mathrm{SMK}-1}$ for lifespan regulation by DAF-16. We began by individually knocking down all $\mathrm{PP} 4{ }^{\mathrm{SMK}-1}$ complex subunits by RNAi, either in daf-2 mutant animals, which are long-lived due to constitutive DAF-16 activation, or in daf-2; daf-16 mutant animals, where this DAF-16-driven effect is absent. Consistent with SMK-1 acting as part of PP4 $4^{\mathrm{SMK}-1}, s m k-1$ as well as $p p h-4.1, p p h-$ 4.2 and $p p f r-2$ were all required for full lifespan extension in daf-2 mutant animals (Fig. 2a). Importantly, their influence on lifespan was completely dependent on the presence of daf-16 (Fig. 2b). Next, we wanted to compare the ability of PP4 $4^{\mathrm{SMK}-1}$ versus other PP4 complexes to promote DAF-16 functions. For this we 
a

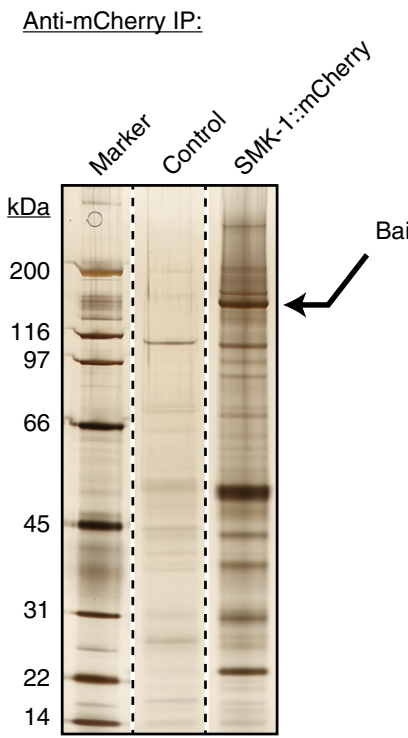

b MS/MS analysis:

\begin{tabular}{|c|c|c|c|c|c|}
\hline $\begin{array}{l}\text { Protein } \\
\text { name }\end{array}$ & Annotation & $\begin{array}{l}\text { Spectral } \\
\text { counts }\end{array}$ & $\begin{array}{l}\text { Unique } \\
\text { peptides }\end{array}$ & $\begin{array}{l}\text { Coverage } \\
{[\%]}\end{array}$ & $\mathrm{MW}[\mathrm{kDa}]$ \\
\hline SMK-1 & Protein phosphatase 4 regulatory subunit & 169 & 93 & 70.0 & 123 \\
\hline PPFR-2 & Protein phosphatase 4 regulatorysubunit & 130 & 35 & 74.1 & 42 \\
\hline PPH-4.1 & Protein phosphatase 4 catalytic subunit & 61 & 26 & 78.4 & 37 \\
\hline PPH-4.2 & Protein phosphatase 4 catalytic subunit & 14 & 12 & 57.6 & 36 \\
\hline SPD-5 & Coiled-coil protein, spindle formation & 12 & 12 & 13.6 & 135 \\
\hline PAA-1 & Protein phosphatase $2 \mathrm{~A}$ structural subunit & 10 & 10 & 24.7 & 66 \\
\hline CEH-79 & Homeobox protein & 9 & 8 & 32.0 & 51 \\
\hline ZK688.9 & Regulator of protein phosphatase $2 \mathrm{~A}$ & 8 & 8 & 40.6 & 33 \\
\hline Y48G1C.8 & Protein of unknown function & 3 & 2 & 4.0 & 172 \\
\hline PDHA-1 & Pyruvate dehydrogenase $\mathrm{E} 1$, alpha subunit & 3 & 3 & 9.1 & 44 \\
\hline F32B6.4 & Protein of unknown function & 3 & 2 & 10.8 & 25 \\
\hline B0280.7 & Protein of unknown function & 3 & 3 & 14.5 & 39 \\
\hline F58D5.7 & Protein of unknown function & 3 & 3 & 15.9 & 29 \\
\hline SPT-5 & DSIF complex subunit, transcriptional & 2 & 2 & 1.9 & 133 \\
\hline RPT-2 & $26 \mathrm{~S}$ proteasome subunit & 2 & 2 & 5.9 & 50 \\
\hline MADF-5 & Transcription factor & 2 & 2 & 6.9 & 48 \\
\hline C24A11.1 & Protein of unknown function & 2 & 2 & 17.3 & 22 \\
\hline
\end{tabular}

d

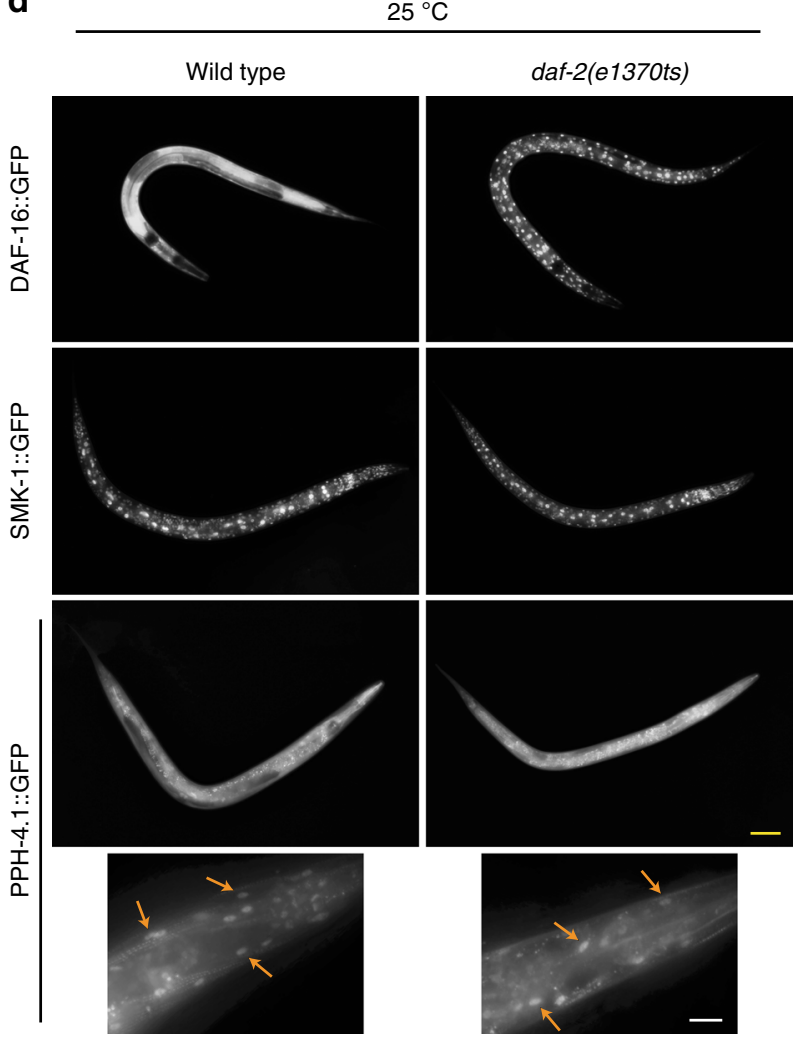

Fig. 1 SMK-1 is part of a globally expressed nuclear Protein Phosphatase 4 complex. a SDS-PAGE and silver staining analysis of an anti-mCherry immunoprecipitation from whole-animal lysates of $C$. elegans expressing SMK-1::mCherry. Animals expressing no transgene were used as negative control. b List of the proteins identified in the purification from $\mathbf{a}$, as determined by mass spectrometry (LC-MS/MS). Only proteins identified by at least two unique peptides are shown. See also Supplementary Table 2 for an independent repeat of this experiment. c Model illustrating the composition of the specific Protein Phosphatase 4 complex (PP4 $4^{\mathrm{SMK}-1}$ ) that co-purified with SMK-1, next to other implicated PP4 subunits not found in this complex. d Localization studies of DAF-16::GFP, SMK-1::GFP, and PPH-4.1::GFP in wild type and in daf-2(e1370ts) mutant animals. Worms were grown from the $\mathrm{L} 1 \mathrm{stage}$ at $15^{\circ} \mathrm{C}$, then shifted to $25^{\circ} \mathrm{C}$ at the L2/L3 stage. After $16 \mathrm{~h}$ the GFP signal was recorded in L4 animals (yellow scale bar: $100 \mu \mathrm{m}$ ). The higher magnification images for PPH-4.1::GFP show head regions. Arrows are highlighting the partial nuclear accumulation of PPH-4.1 in both wild type and daf-2 (e1370ts) animals (white scale bar: $20 \mu \mathrm{m}$ ).

extended the previous experiment to include knockdowns of ppfr-1 and ppfr-4-two PP4 subunits that we found to be absent from $\mathrm{PP}^{\mathrm{SMK}-1}$ (Fig. 1b, c). Consistent with $\mathrm{PP} 4^{\mathrm{SMK}-1}$ being the preferred PP4 configuration to regulate DAF-16 functions, loss of $p p f r-1$ had no effect on lifespan (Supplementary Fig. 2a, b).
And although loss of ppfr-4 shortened the lifespan of daf-2 animals, it did so in a manner independent of daf-16 (Supplementary Fig. 2a, b).

In Fig. 2a, RNAi of pph-4.1 or pph-4.2 had a lesser effect on DAF-16-driven longevity than RNAi of $s m k-1$. We attributed this 

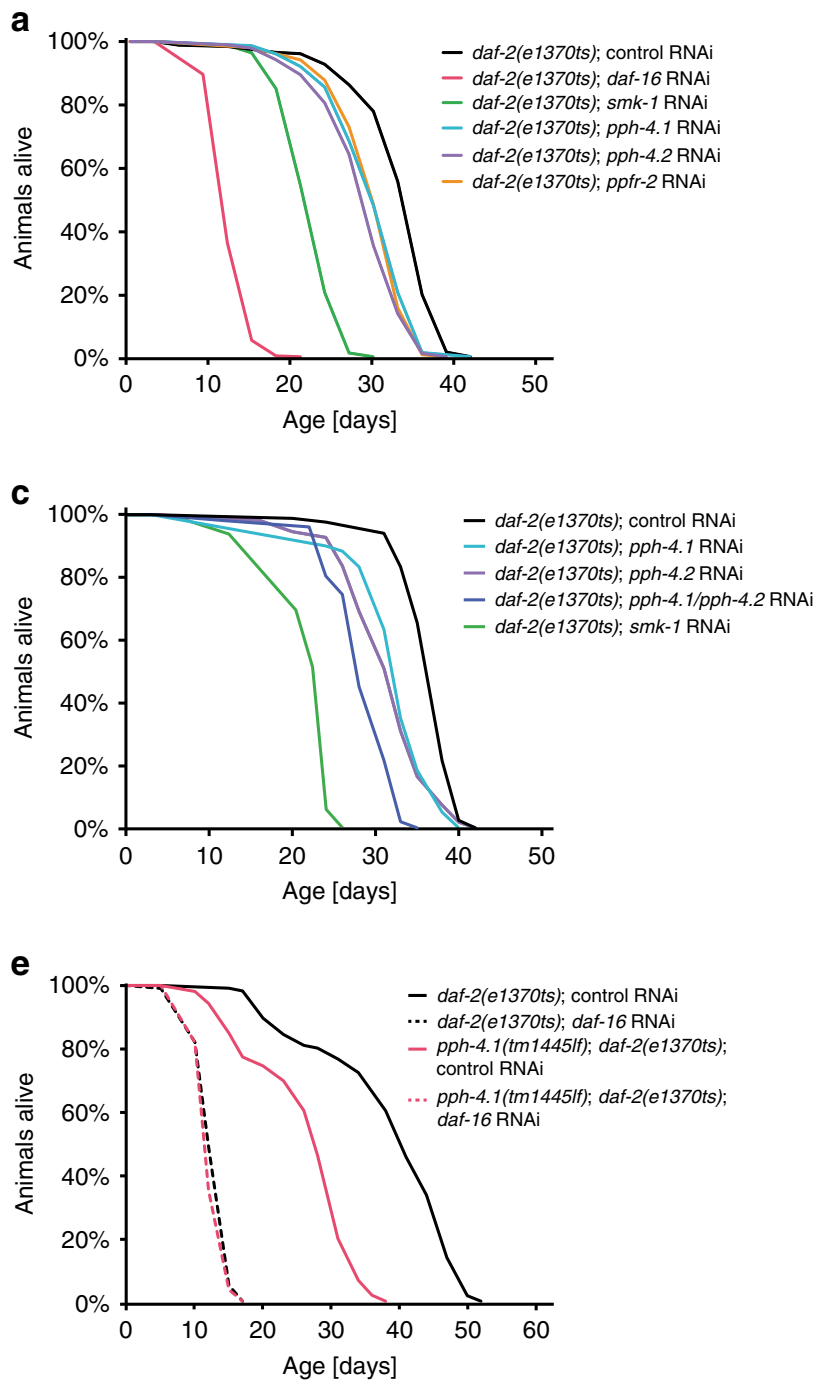
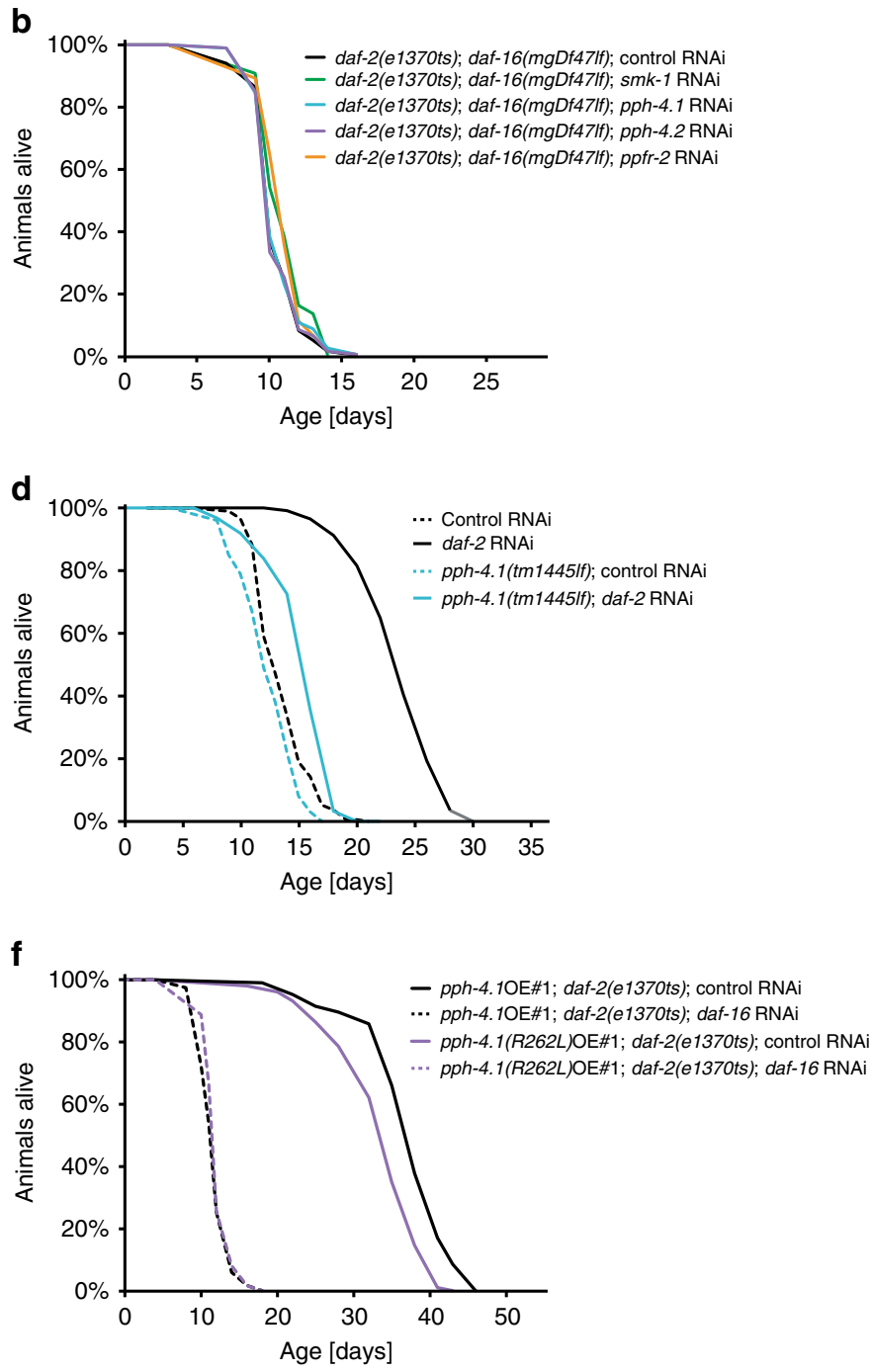

Fig. 2 SMK-1 promotes the DAF-16-mediated longevity induced by low IIS as part of PP4 ${ }^{\text {SMK-1. }}$. Lifespan phenotypes caused by loss of daf-16 or specific PP4 subunits in various genetic backgrounds. Animals of indicated genotypes were grown from the L1 stage on the indicated RNAi bacteria at $15^{\circ} \mathrm{C}$. At the L4 stage the animals were shifted to $25^{\circ} \mathrm{C}$ to fully inactivate daf-2(e1370ts) and their lifespan was monitored. All strains used in a-c harbored the eri- 1 (mg366ts) mutation to yield better knockdown efficiencies ${ }^{51}$. a Individual knockdown of daf- 16 or any of the PP4SMK-1 subunits shortened the lifespan of daf-2(e1370ts) mutant animals. b All these phenotypes were abolished in daf-16(mgDf47lf) null mutant animals. c Combined knockdown of the catalytic PP4SMK-1 subunit paralogs pph-4.1 and pph-4.2 enhanced the lifespan-shortening effects of their individual knockdowns in daf-2(e1370ts) mutant animals. d, e The pph-4.1(tm1445If) null mutation strongly impaired the longevity of low IIS animals in a daf-16-dependent manner, too. $\mathbf{f}$ Lifespan analyses of daf-2 (e1370ts) mutant animals ectopically expressing either catalytically active PPH-4.1 or catalytic dead PPH-4.1(R262L). Expression of the catalytically dead PPH-4.1(R262L) specifically impaired the longevity of daf-2(e1370ts) mutant animals. For detailed statistics see Supplementary Table 4. Source data are provided as a Source Data file.

to a possible redundancy of these two PP4 catalytic subunits. To test this, we conducted double-RNAi experiments targeting $p p h$ 4.1 and $p p h-4.2$ together. Indeed, combined knockdown of both catalytic subunits led to additive suppression of longevity in daf-2 animals, to an extent now more comparable to smk-1 RNAi (Fig. 2c). Again, these lifespan phenotypes were dependent on daf-16, as illustrated by conducting the same knockdowns in wild type (Supplementary Fig. 2c) or daf-2; daf-16 mutant animals (Supplementary Fig. 2d).

We then validated the phenotypes obtained from knockdown of the PP4 $4^{\mathrm{SMK}-1}$ catalytic subunits using a loss-of-function allele of pph-4.1, pph-4.1(tm1445). This allele lacks exons 3 and 4 of $p p h-4.1$, which contain the active site of the enzyme. Consistent with our RNAi data, this allele also specifically suppressed the longevity of daf-2 mutant animals, while it had little effect in wild type and no effect in daf-2; daf-16 mutant animals (Fig. 2d, e).
So far, our data was consistent with SMK-1 and PPH-4.1/4.2 each affecting lifespan as part of the PP4 $4^{\mathrm{SMK}-1}$ complex, acting through the same genetic pathway. If this were true, then combined depletion of $s m k-1$ and $p p h-4.1 / 4.2$ should lead to largely nonadditive phenotypes. We tested this by lifespan assays in daf-2 mutant animals, confirming that this was indeed the case (Supplementary Fig. 2e).

Finally, we tested if it is truly the catalytic activity rather than structural properties of $\mathrm{PP} 4 \mathrm{SMK}-1$ that promote DAF-16 functions. We created transgenic lines overexpressing either wild type pph-4.1 or an allele encoding an R262L mutant form of PPH-4.1, which has been shown to be catalytically inactive by studies in $C$. elegans and mammals ${ }^{14,15}$. Consistent with the importance of the catalytic activity of PP4 $4^{\mathrm{SMK}-1}$, overexpression of the catalytic dead form of PPH-4.1 led to partial suppression of longevity in daf-2 mutant animals (Fig. 2f, Supplementary Fig. 2f), and this lifespan- 
shortening effect was lost upon knockdown of daf-16 (Fig. 2f, Supplementary Fig. 2f).

We conclude that SMK-1 acts as part of a specific PP4 complex and uses its catalytic activity to promote DAF-16 functions, as shown here for DAF-16-driven longevity under low IIS.

\section{PP4 ${ }^{\text {SMK-1 }}$ promotes only some DAF-16-driven stress responses.} Much of the DAF-16-driven longevity in daf-2 mutant animals can be attributed to improved stress responses ${ }^{6}$. Consistently, a previous study had shown that SMK-1 was required for the daf16-dependent improved resistance of daf-2 animals to oxidative stress and UV irradiation ${ }^{8}$. Notably though, SMK-1 showed surprising specificity in mediating these phenotypes, as it did not affect the daf-16-dependent increased resistance to for example heat stress in these animals ${ }^{8}$. We now tested, if PP4 $4^{\mathrm{SMK}-1}$ confers stress resistance with similar specificity. For this we knocked down the catalytic subunits, $p p h-4.1$ and $p p h-4.2$, by combined RNAi in either daf-2 or daf-2; daf-16 mutant animals and exposed them to either $1500 \mathrm{~J} / \mathrm{m}^{2} \mathrm{UV}$ irradiation or shifted them to $6 \mathrm{mM}$ tert-butyl hydroperoxide $(\mathrm{tBOOH}$; oxidative stress) or to $32^{\circ} \mathrm{C}$ (heat stress) at day 2 of adulthood. Survival under these stresses was determined using a lifespan scoring machine ${ }^{16}$, to obtain data of particularly high resolution. We found that just like RNAi against smk-1, RNAi against pph-4.1/ pph-4.2 also led to reduced resistance to $\mathrm{tBOOH}$ and UV irradiation but not to heat (Fig. 3a, c, e). Furthermore, consistent with these being daf-16-dependent effects, these phenotypes were reduced in wild type animals when daf-16 activity is reduced (Supplementary Fig. 3), and were entirely absent in daf-16 null mutant animals (Fig. 3b, d, f).

These data illustrate that SMK-1's roles in promoting stress resistance under low IIS also rely on the catalytic subunits of PP4 ${ }^{\text {SMK- }}$; and we could confirm the prior observation that SMK1 , and now PP4 ${ }^{\mathrm{SMK}-1}$, selectively help DAF-16 to confer a subset of its stress responses, e.g. resistance to oxidative stress and UV but not heat stress.

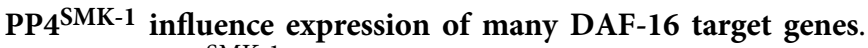
Given that PP4 ${ }^{\mathrm{SMK}-1}$ is required for many of DAF-16's functions under low IIS, and because DAF-16 is a transcription factor, we wondered whether $\mathrm{PP} 4^{\mathrm{SMK}-1}$ acts by influencing the expression of DAF-16-regulated genes. We conducted mRNA-seq experiments using either wild type or daf-2 mutant $C$. elegans. The animals were treated with either control, daf-16, or smk-1 RNAi from the L1 stage and their mRNA sequenced at young adulthood. From the resulting dataset, we first determined the genes differentially expressed by the reduced IIS in daf-2 mutant animals, identifying 1093 upregulated and 2054 downregulated genes (Fig. 4a). These gene expression changes were consistent with previous work ${ }^{17}$ (Supplementary Table 9). Next we asked how these differentially expressed genes are affected by RNAi against daf-16 or $s m k-1$ in daf-2 mutant animals. Most of the gene expression changes that occur in daf- 2 mutant animals are known to be mediated by DAF-16 6,17 . In agreement with this prior work, RNAi of daf-16 suppressed $62.1 \%$ of the gene activation and $87.1 \%$ of the gene repression events in daf-2 mutant animals (Fig. 4a, Supplementary Table 9). RNAi of $s m k-1$ also suppressed many of these gene expression changes, namely $40.7 \%$ and $18.7 \%$, respectively (Fig. 4a). These findings were consistent with $\mathrm{PP} 4{ }^{\mathrm{SMK}-1}$ indeed influencing the lifespan extending and largely DAF-16-induced gene expression changes under low IIS. Next we determined which actual genes were regulated by DAF-16 or SMK-1 under low IIS and which genes were common targets of both. As expected from the results in Fig. 4a, we found significant overlap amongst the genes that were activated or amongst the genes that were repressed by DAF-16 and SMK-1 $\left(p=4.95 \times 10^{-22}\right.$ and $p=$ $7.78 \times 10^{-51}$, respectively; Fig. $\left.4 \mathrm{~b}, \mathrm{c}\right)$. And consistent with both DAF-16 and SMK-1 being required for the longevity under low IIS, we found these co-regulated genes to be enriched for genes related to aging (based on GO-term enrichment analyses, Fig. 4d, e).

Taken together, $\mathrm{PP} 4^{\mathrm{SMK}-1}$ is required for DAF-16 to properly regulate a subset of its target genes-many of which are aging related.

PP4 ${ }^{\text {SMK-1 }}$ barely affects DAF-16 activation and DNA binding. Having established that PP4 $4^{\text {SMK-1 }}$ is important for many DAF-16 functions under low IIS, presumably by helping it to regulate aging-related target genes, we wondered how this function arises. Between the initial DAF-16-activating stimulus of low IIS activity and the successful regulation of target genes resides a long series of events. At which level of this cascade does PP4 $4^{\text {SMK-1 }}$ act? First, we studied the kinetics of nuclear entry and exit of DAF-16 upon inactivation and reactivation of the temperature-sensitive daf-2 (e1370) allele, respectively (Supplementary Fig. 4a). Previous work only comparing translocation endpoints had found no impact of SMK-1 on the nuclear translocation of DAF- $16^{8}$. Consistently, we observed no difference in the endpoints of our nuclear entry and nuclear exit time courses (Supplementary Figs. 4b, c, and 5). Only when we followed the kinetics of nuclear translocation we observed that knockdown of $s m k-1$ led to a mild delay, particularly in the entry of DAF-16 upon daf-2 inactivation (Supplementary Figs. 4b, c and 5). However, we interpret this phenotype as very moderate and hence unlikely to explain the strong impact of $\mathrm{PP} 4{ }^{\mathrm{SMK}-1}$ on DAF-16 functions.

Upon nuclear entry of DAF-16, it next needs to bind to its target promoters (Supplementary Fig. 4a). To see if this is influenced by $\mathrm{PP} 4^{\mathrm{SMK}-1}$ we determined the genome-wide binding of DAF-16 to its target promoters in the presence or absence of SMK-1. We used daf-2 mutant animals expressing DAF-16::GFP, treated them with either control or smk-1 RNAi, and then subjected them to chromatin immunoprecipitation (ChIP)-seq analysis using an anti-GFP antibody. Looking across 2824 DAF16 binding sites described in previous work ${ }^{18}$, we found no significant change in the binding of DAF-16 to its target regions ( $p=0.152$; Supplementary Fig. 4d; see also Supplementary Fig. 4e for examples of individual genes contributing to Supplementary Fig. 4d).

In summary, absence of SMK-1 led to only a slight delay in DAF-16 nuclear entry and no significant change in the binding of DAF-16 to its target promoters. We therefore conclude that

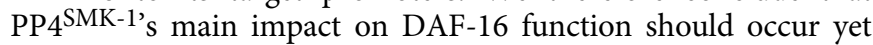
further downstream.

PP4 ${ }^{\text {SMK-1 }}$ promotes transcription initiating events. DAF-16 is predominantly a transcriptional activator ${ }^{17}$. Thus, upon binding its target promoters the next important downstream steps should be: (1) the recruitment of RNA Polymerase II (Pol II) to the promoters; (2) promoter clearance, which causes Pol II to leave the promoter region and move into the gene body where it often will pause around the +50 position (promoter-proximal pausing $)^{19,20}$; (3) transcriptional elongation, during which Pol II moves beyond the pause site and transcribes the entire gene (Fig. 5a). To evaluate these steps we used daf-2 mutant animals, subjected them to either control or smk-1 RNAi, and then determined the genome-wide distribution of Pol II on chromatin by ChIP-seq using an antibody against the large subunit of Pol II, AMA-1. Strikingly, while Pol II recruitment was unaffected by $s m k-1$ RNAi when looking cumulatively at all promoter regions genome-wide $(p=0.796)$, we saw a substantial reduction in Pol II 

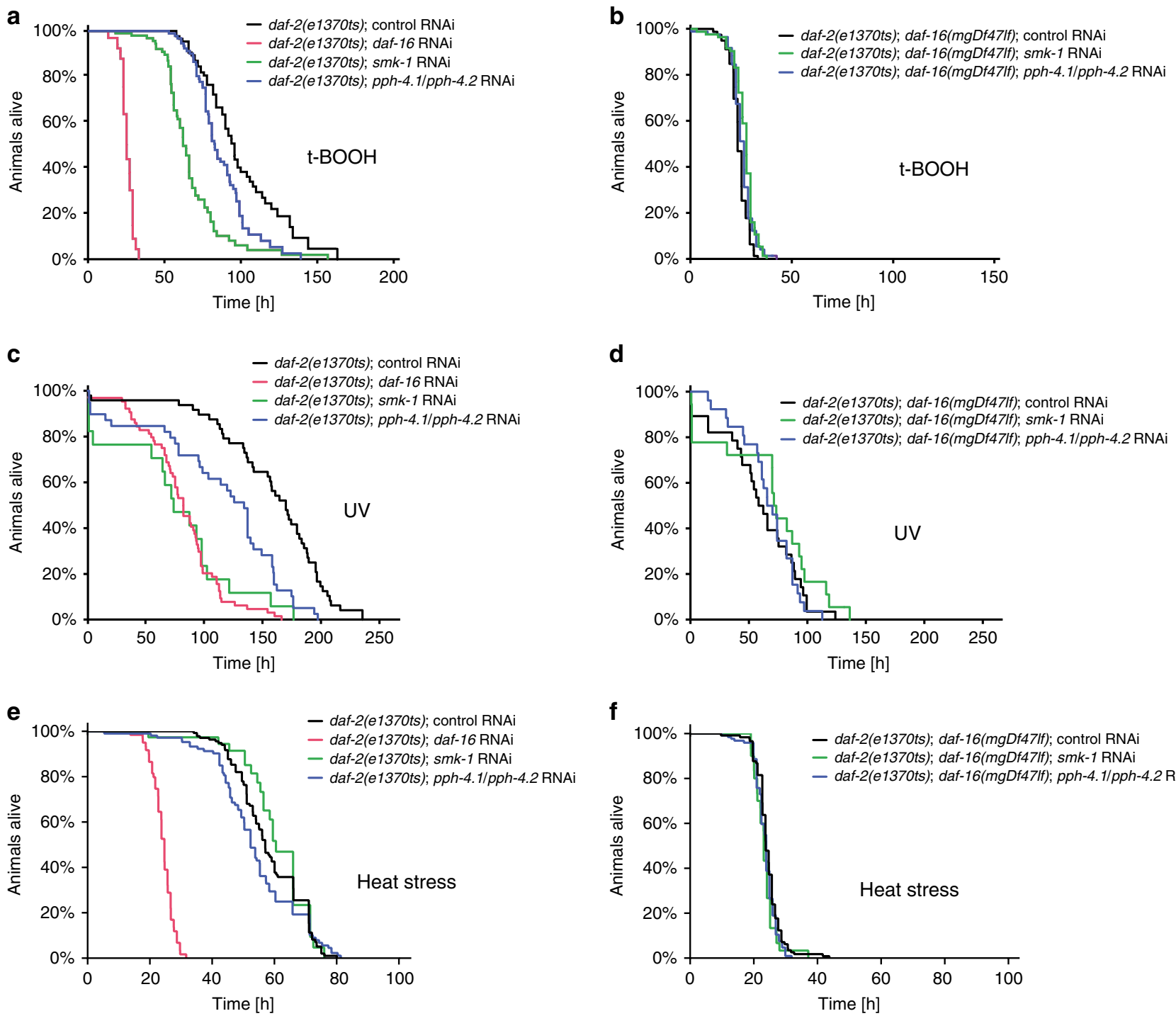

Fig. 3 Under low IIS, PP4 ${ }^{\text {SMK-1 }}$ is required for DAF-16-mediated resistance to oxidative stress and UV. Stress survival phenotypes caused by loss of daf-16 or PP4 $4^{\mathrm{SMK}-1}$ in various genetic backgrounds. Animals of indicated genotypes were grown at $15^{\circ} \mathrm{C}$ from the L1 stage on the indicated RNAi bacteria. At the $\mathrm{L} 4$ stage the temperature was shifted to $25^{\circ} \mathrm{C}$ to fully inactivate daf-2(e1370ts). On day 2 adulthood, animals were then transferred to $6 \mathrm{mM}$ tBOOH containing RNAi plates (oxidative stress), exposed to $1500 \mathrm{~J} / \mathrm{m}^{2}$ UV light (UV stress), or shifted to $32{ }^{\circ} \mathrm{C}$ (heat stress). All strains used in in this figure harbored the eri-1(mg366ts) mutation to yield better knockdown efficiencies ${ }^{51}$. a, c Loss of PP4SMK-1, either by knockdown of SMK-1 or double knockdown of PPH-4.1/PPH-4.2, impaired the enhanced survival of daf-2(e1370ts) mutant animals that were exposed to oxidative stress (a) or UV stress (c). $\mathbf{b}$, $\mathbf{d}$ These phenotypes were abolished in the absence of daf-16. e, $\mathbf{f}$ In contrast to oxidative stress and UV stress, loss of these PP4 ${ }^{\text {SMK-1 }}$ subunits did not impair the enhanced survival of daf-2(e1370ts) mutant animals exposed to heat stress. For detailed statistics see Supplementary Table 4. Source data are provided as a Source Data file.

recruitment to promoters whose downstream genes would both be activated by DAF-16 and depend on SMK-1 for their activation (genes co-activated by DAF-16 and SMK-1 according to Fig. $4 \mathrm{~b})(p=0.012$; Fig. $5 \mathrm{~b}$, see left panels of Supplementary Fig. 6 for examples of genes that exhibit this phenotype).

Besides this apparent phenotype, we also noted an unexpected distribution of Pol II at these promoters. When we looked cumulatively at the regions around transcriptional start sites (TSSs) genome-wide, most Pol II had already undergone transcriptional initiation and localized to the pause site in the gene body (Fig. 5c). A similar distribution of Pol II could be found when looking cumulatively at all genes activated by DAF16 (Fig. 5c). However, at the genes co-activated by DAF-16 and SMK-1, the distribution of Pol II was noticeably wider and a substantial amount of Pol II localized upstream of the TSS
(Fig. 5c, see red arrow). We believe that this Pol II has been recruited to promoters but not fully undergone promoter clearance yet, which may indicate that the events of transcriptional initiation at DAF-16/SMK-1-co-activated genes occur slower and hence may be particularly rate limiting for their transcription $^{21}$. Upon $s m k-1$ RNAi, presence of Pol II upstream of the TSS was even more pronounced (Fig. 5b), which would be consistent with SMK-1 promoting not only Pol II recruitment but also promoter clearance. However, we remain uncertain of this phenotype as it was difficult to recapitulate on the level of individual genes (see right panels of Supplementary Fig. 6 for example genes).

To further test our notion of transcription initiating events being impaired, we repeated our ChIP-seq experiments using an antibody that detects only initiating Pol II, which is 
a

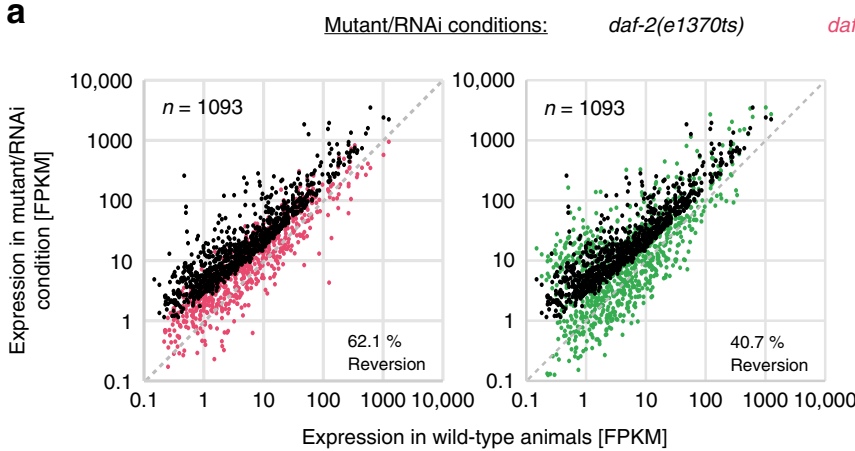

daf-2(e1370ts); daf-16(RNAi) daf-2(e1370ts); smk-1(RNAi)

b
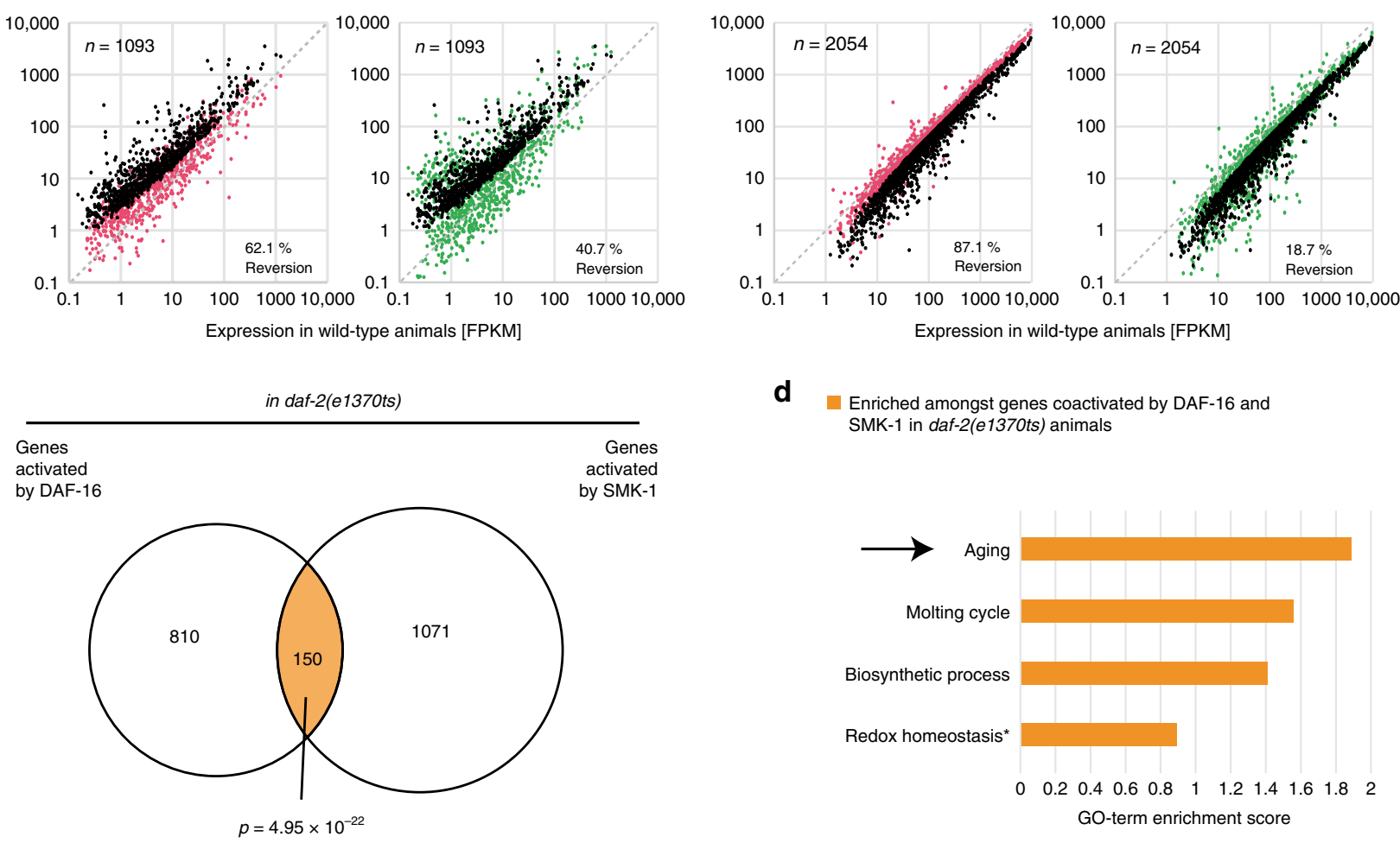

d Enriched amongst genes coactivated by DAF-16 and
SMK-1 in daf-2(e1370ts) animals

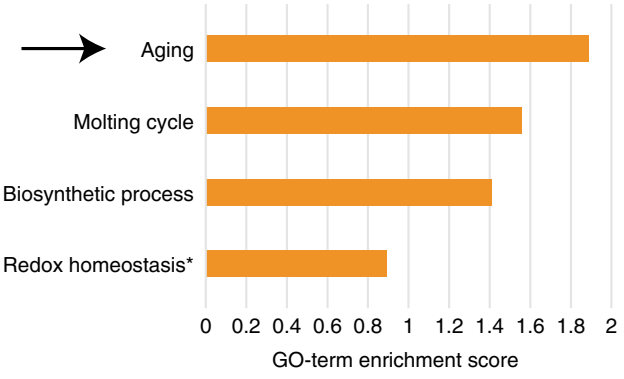

C

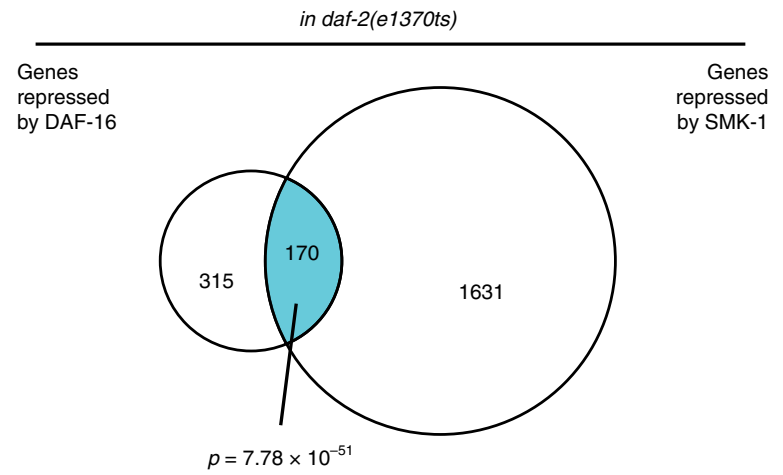

e

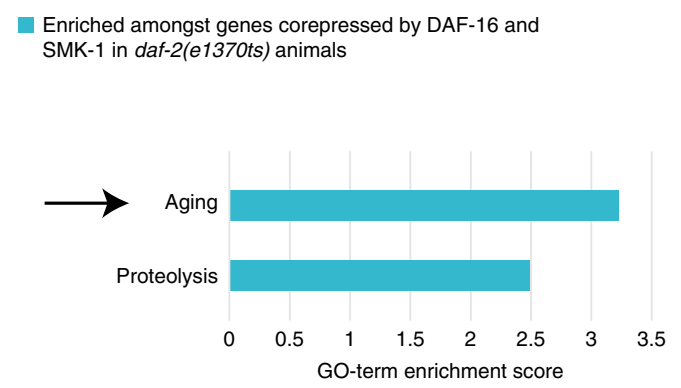

Fig. 4 PP4 ${ }^{\text {SMK-1 }}$ is required for a substantial part of the DAF-16-mediated gene expression changes induced by low IIS. daf-2(e1370ts) mutant C. elegans were grown from the L1 stage on either control, daf-16, or smk-1 RNAi bacteria. At the L4 stage, animals were shifted for $16 \mathrm{~h}$ to $25^{\circ} \mathrm{C}$, then harvested, and their transcriptomes determined by mRNA-seq. All strains used in in this figure harbored the eri-1(mg366ts) mutation to yield better knockdown efficiencies ${ }^{51}$. a Similar to daf- 16 loss, smk-1 loss also suppresses many of the gene expression changes that occur under low IIS. The scatter plots on the left show the genes significantly upregulated and the scatter plots on the right show the genes significantly downregulated in daf-2(e1370ts) compared with wild type animals. b, c Venn diagrams illustrate the number of genes significantly regulated by DAF-16 or SMK-1 in daf-2(e1370ts) mutant animals as well as their overlap. d, e Functional enrichment analyses, conducted on the co-activated and co-repressed genes shown in $\mathbf{b}$ and $\mathbf{c}$. Only significant enrichments are shown (DAVID scores $\geq 1.3$, the symbol asterisk indicates that this term was still amongst the four most enriched GO-terms and relevant in the context of aging and stress responses, but it had a score below the significance threshold). Arrows highlight the GO-term "aging", which turned out to be the most enriched in both analyses.

phosphorylated at the Ser5 position within the repeats of its Cterminal domain (CTD) ${ }^{22,23}$. Indeed, we found significantly less initiating Pol II in the TSS regions of DAF-16/SMK-1-coactivated genes (Fig. 5d).

Finally, we investigated if $\mathrm{PP} 4^{\mathrm{SMK}-1}$ influences $\mathrm{Pol}$ II pausing, pause release or transcriptional elongation. A good indicator for defects in these processes are changes in the pausing index (the ratio of the average read densities of paused to elongating Pol II), as it can be derived from Pol II ChIP-seq data ${ }^{24}$. Calculating this index for DAF-16/SMK-1-co-activated genes under low IIS we found no impact of $s m k-1$ RNAi on the pausing index (Supplementary Table 6), suggesting that PP4 ${ }^{\mathrm{SMK}-1}$ had no direct role in Pol II pausing, pause release or transcriptional elongation.

We conclude that $\mathrm{PP} 4^{\mathrm{SMK}-1}$ is required for transcriptional initiation, predominantly by promoting efficient Pol II recruitment at a subset of DAF-16-activated target genes, and that these are genes whose expression may particularly depend on transcriptional initiation as a rate limiting step. We propose that this function is the major reason why $\mathrm{PP} 4^{\mathrm{SMK}-1}$ is required for the induction of many DAF-16 target genes under low IIS. 
a

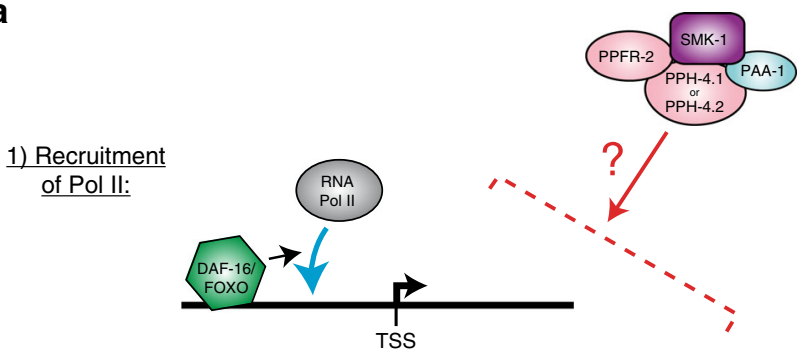

2) Promoter Clearance:

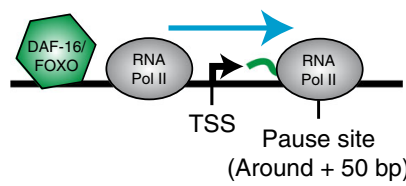

3) Transcriptional elongation:

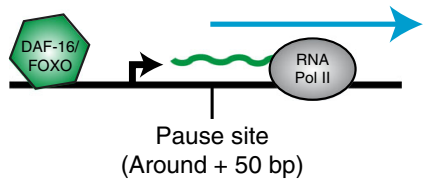

C

ChIIP of RNA Pollll in daf-2 $(\underline{e} 1 \underline{37} \underline{0 t} \underline{s}):$

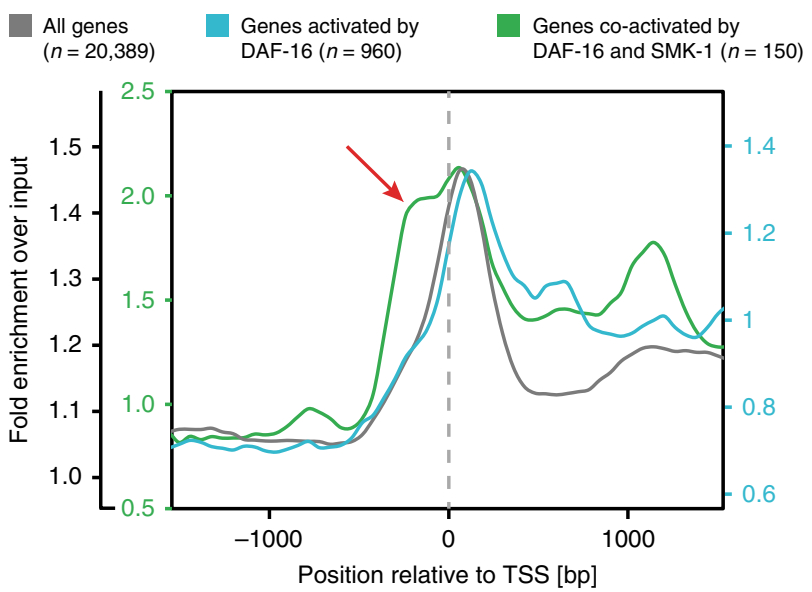

b

ChIP of RNA Pol Il in daf-2(e1370ts):

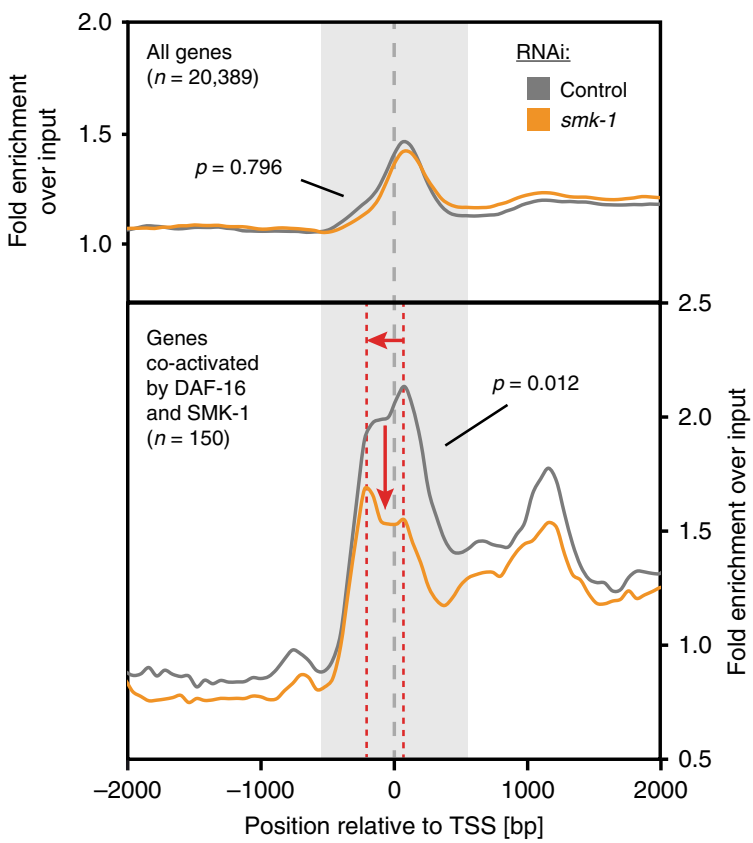

d

ChIP of RNA Pol II(Ser5Phos) in daf-2(e1 $\underline{370 t s)}$ :

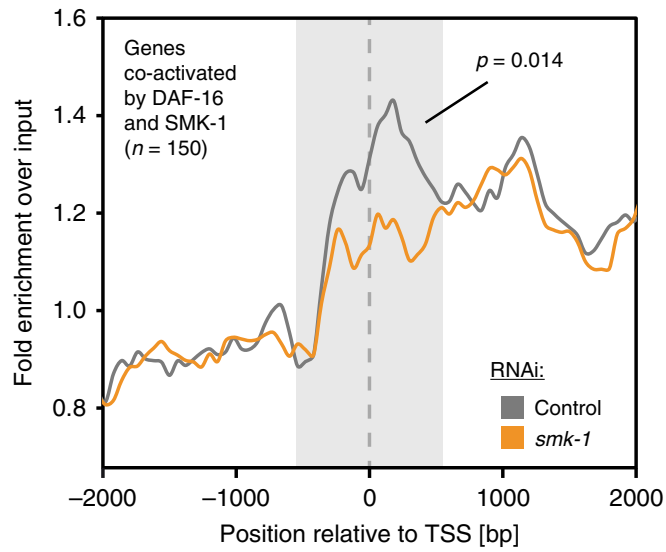

Fig. 5 PP4 ${ }^{\text {SMK-1 }}$ is required for transcription initiating events at the genes co-activated by DAF-16 and SMK-1. a Schematic of the key steps that lead from DAF-16's binding to promoter regions to the eventual transcription of the downstream genes. b-d ChIP-seq analysis of RNA Pol II under low IIS. daf-2 (e1370ts) mutant animals were synchronized and grown from the L1 stage on either control or smk-1 RNAi bacteria. At the L4 stage, animals were shifted to $25^{\circ} \mathrm{C}$. After an additional $16 \mathrm{~h}$, animals were harvested and ChIP-seq analysis was conducted, using antibodies recognizing RNA Pol II, either in any modification state $(\mathbf{b}, \mathbf{c})$ or specifically RNA Pol II that was phosphorylated at Ser5 of its CTD (d). Average read densities across TSS regions of the indicated gene sets are shown. $p$ values indicate the significance of read density differences in the grayed regions ( -600 to +600 around the TSSs) between control and smk-1 RNAi treated animals. Source data underlying Fig. 5b, d are provided as a Source Data file.

The relevant substrate of PP4 $4^{\text {SMK-1 }}$ is SPT-5. We now had identified the steps in the cascade from DAF-16 activation to target gene expression which depend on $\mathrm{PP} 4{ }^{\mathrm{SMK}-1}$. But $\mathrm{PP} 4 \mathrm{SMK}-1$ is a phosphatase, and we still did not know the relevant substrate whose dephosphorylation influences Pol II recruitment and behavior at a subset of DAF-16 target genes. First we took a candidate approach to test whether DAF-16 itself could be the target. However, neither our IP-MS/MS experiments (Fig. 1a, b, Supplementary Tables 2, 3, and ref. ${ }^{17}$ ) nor published co-IPs ${ }^{8}$ provided evidence for a physical interaction between $\mathrm{PP} 4^{\mathrm{SMK}-1}$ and DAF-16. In addition, we investigated the smk-1-dependent phosphorylation status of DAF-16 by phos-tag gel- electrophoresis. We grew DAF-16::GFP-expressing C. elegans containing either daf-2(e1370) or daf-18(mg198) and exposed them to either control or $s m k-1$ by RNAi. As expected from high IIS activity leading to phosphorylation of DAF-16, we observed an upshift of DAF-16::GFP in daf-18 versus daf-2 mutant protein extracts when analyzed by a phos-tag gel and western blotting, but RNAi of $s m k-1$ had no effect on the migratory behavior of DAF-16::GFP in this assay (Supplementary Fig. 7). All this data argued that the relevant substrate of $\mathrm{PP} 4^{\mathrm{SMK}-1}$ is distinct from DAF-16.

To identify the relevant substrate, we turned to an unbiased phosphoproteomics approach. We grew daf-2 mutant animals to 
a

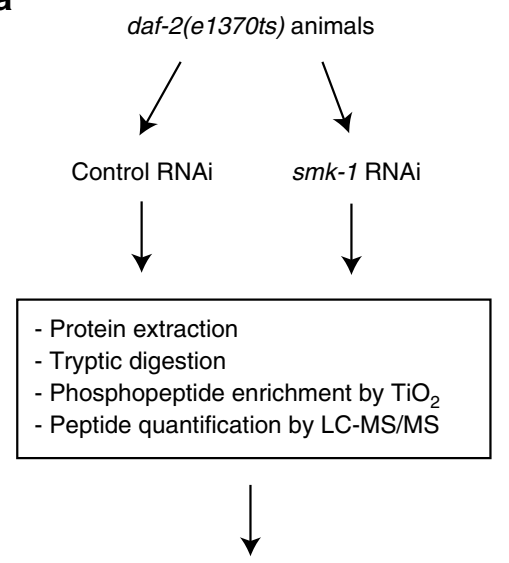

208 phosphopeptides (derived from 150 proteins) showed increased abundance under smk-1 RNAi

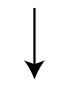

41 phosphoproteins were selected as candidates for further evaluation

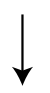

RNAi screen for candidates influencing expression of the DAF-16 target sod-3

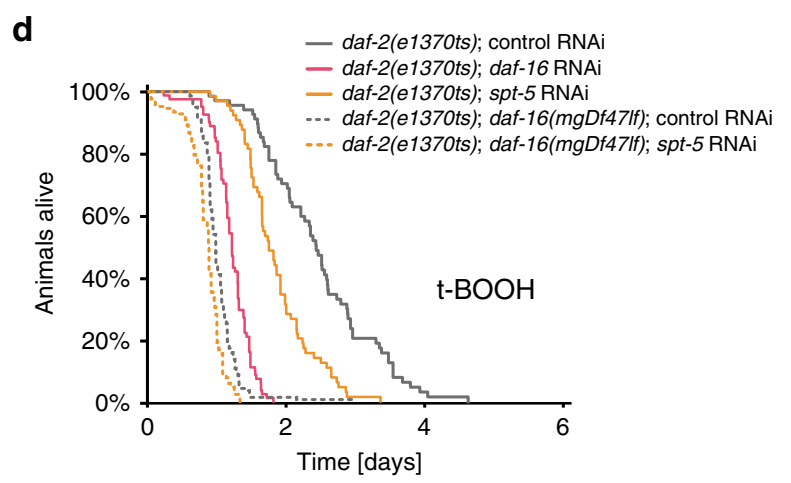

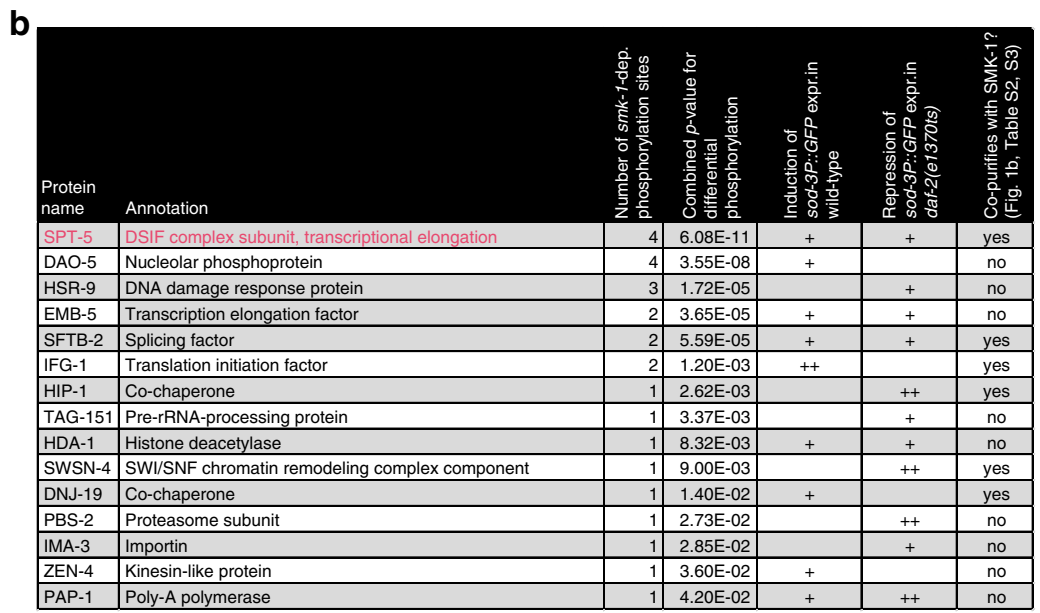
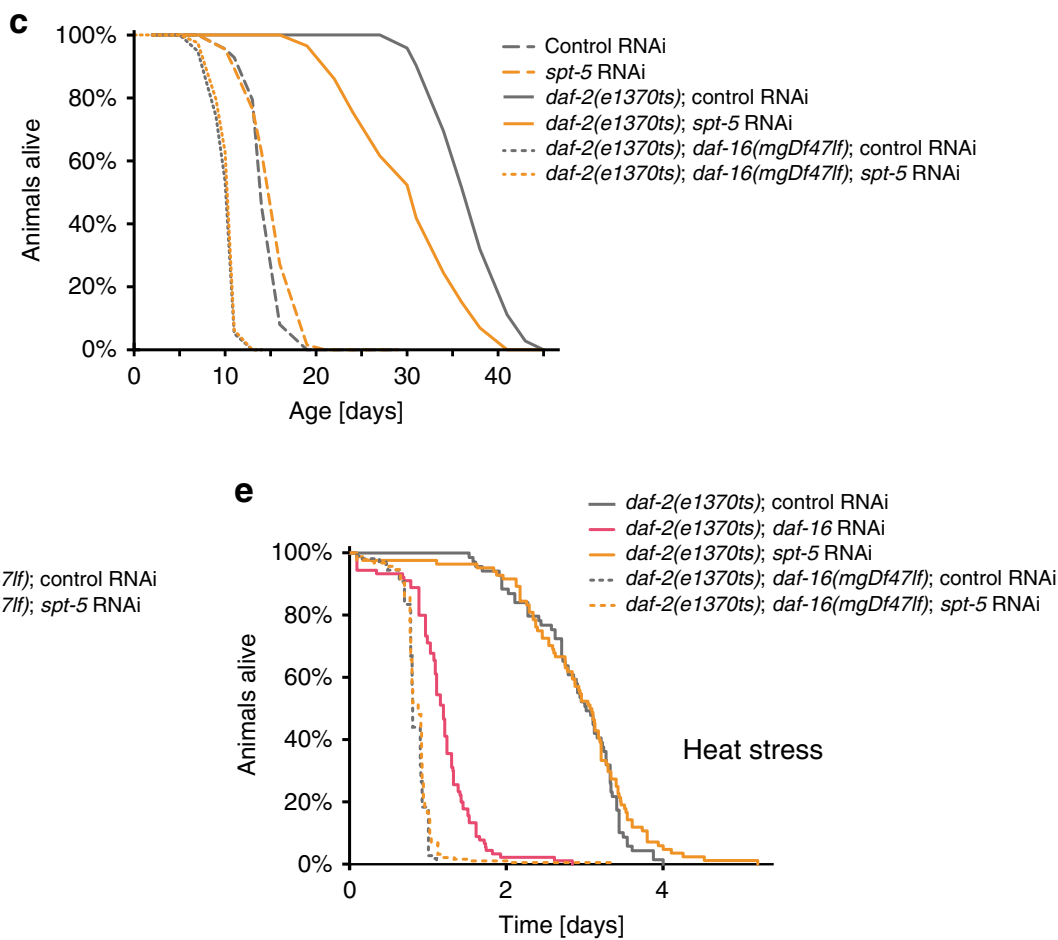

Fig. 6 PP4 ${ }^{\text {SMK-1 }}$ promotes DAF-16's functions under low IIS by dephosphorylating SPT-5. Identification of the relevant PP4 ${ }^{\text {SMK-1 }}$ substrate by unbiased phosphoproteomics and functional evaluation of the emerging candidates. a Workflow of the phosphoproteomics approach to identify PP4 ${ }^{\text {SMK-1 }}$ substrate candidates, followed by their functional evaluation through RNAi screening using a sod-3P::GFP reporter in either wild type or daf-2(e1370ts) backgrounds. b Results from the approach shown under $\mathbf{a}$. The table is limited to proteins that were found significantly more phosphorylated upon smk-1 RNAi and that additionally affected expression of sod-3, i.e., by sod-3 induction in wild type or the repression of sod-3 expression in daf-2(e1370ts) mutant animals. The table is ranked by the significance ( $p$ value) of the increased phosphorylation observed upon smk-1 RNAi. SPT-5 is highlighted in red as being the topranked candidate from this prioritization approach. c-e Loss of SPT-5 phenocopied the stress resistance and lifespan phenotypes caused by loss of SMK-1. All strains used in in this figure harbored the eri-1(mg366ts) mutation to yield better knockdown efficiencies ${ }^{51}$. Wild type, daf-2(e1370ts), or daf-16 (mgDf47lf); daf-2(e1370ts) mutant animals were grown from the L4 stage on either control or spt-5 RNAi bacteria at $25^{\circ} \mathrm{C}$. Animals remained either untreated (c), or were grown until day 2 of adulthood and then transferred to either $6 \mathrm{mM} \mathrm{tBOOH}$ containing RNAi plates (oxidative stress) (d) or shifted to $32{ }^{\circ} \mathrm{C}$ (heat stress) (e). Survival of the animals was monitored. For detailed statistics see Supplementary Table 4. Source data underlying Fig. $6 \mathrm{c}-\mathrm{e}$ are provided as a Source Data file.

scale, treated them with either control or smk-1 RNAi, lysed them, and determined their phosphoproteome by $\mathrm{TiO}_{2}$-based phosphopeptide enrichment and LC-MS/MS (see also Fig. 6a). 208 phosphopeptides whose abundance increased upon smk-1 $\mathrm{RNAi}-$ and thus should be direct or indirect targets of PP4 $4^{\mathrm{SMK}-1}$ - could be identified (Fig. 6a, Supplementary Table 7). The phosphopeptides originated from 150 proteins (Supplementary Table 7), of which we selected 41 for further evaluation based on their annotation and the significance of their smk-1-dependent differential phosphorylation. To determine the functional relevance of these candidate proteins, we used a transcriptional reporter for the canonical DAF-16 target gene sod-3. This reporter is induced by DAF-16 under low IIS-an induction that requires $\mathrm{SMK}-1^{8}$. Hence we knocked down each of the 41 candidates by RNAi in wild type or daf-2 mutant animals carrying Psod-3::GFP and evaluated their GFP expression from 
day 1 to day 3 of adulthood. Knockdown of several candidates led to substantial changes in the expression of this reporter (Fig. 6b, Supplementary Fig. 8a). We additionally searched our SMK-1 IP-MS/MS data to see whether any of these candidates copurified with PP4 ${ }^{\mathrm{SMK}-1}$, a behavior that often can be observed for phosphatase substrates ${ }^{25}$. Indeed, we found that several of our candidate substrates co-purified with SMK-1 (Figs. $1 \mathrm{~b}$ and $6 \mathrm{~b}$, Supplementary Tables 2, 3). Together, these analyses highlighted one candidate in particular-the evolutionarily conserved protein SPT-5, which is predominantly known as a transcriptional elongation factor (Fig. 6b). Firstly, SPT-5 influences the expression of sod-3 (Fig. 6b, Supplementary Fig. 8a). Secondly, SPT-5 harbors four phosphorylation sites that become more phosphorylated in the absence of $\mathrm{PP} 4^{\mathrm{SMK}-1}$ under low IIS (Fig. 6b, Supplementary Table 7). This differential phosphorylation of SPT-5 had the best $p$ value of all sod-3-influencing candidates. Thirdly, SPT-5 physically associated with PP4SMK-1 according to our IP-MS/MS data (Figs. $1 \mathrm{~b}$ and $6 \mathrm{~b}$, Supplementary Table 3)-an interaction that we were able to validate even between the human orthologs of SMK-1 and SPT-5 in HEK293T cells (Supplementary Fig. 8c). Finally, two additional transcription elongation factors from the same SPT family which closely synergize with SPT $-5^{26}$ emerged from our study: (1) EMB$5^{27}$, which just like SPT- 5 is a candidate substrate of PP4 $4^{\text {SMK- } 1}$ (Fig. 6b); and (2) SPT-4, which just like SPT-5 can be bound by

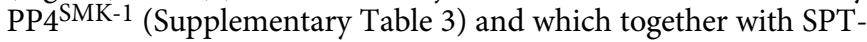
5 forms the so-called DSIF (DRB sensitivity inducing factor) complex, involved in regulating Pol II's promoter-proximal pausing and transcriptional elongation ${ }^{28}$. We then tested the consequences of losing any of these SPT family members for DAF-16-driven longevity and the increased resistance to oxidative and heat stress under low IIS. Already during the sod3 reporter assays we observed that RNAi of $s p t-5$ and $e m b-5$ from the L1 stage leads to developmental arrest. This is not surprising, as they should be essential for transcription throughout the genome $^{24}$. Hence we conducted knockdowns of $s p t-4$ from the L1 or L4 stage, while spt-5 and emb-5 were knocked down from the L4 stage to overcome developmental phenotypes and hopefully leave behind sufficient residual amounts of these proteins to retain a basic level of genome-wide transcription. In these experiments, RNAi of $e m b-5$ or $s p t-4$ did not affect DAF-16driven longevity under low IIS (Supplementary Fig. 8b; L1 feeding data for $s p t-4$ RNAi are not shown), leading us to exclude these SPT family members as relevant substrates. However strikingly, RNAi of $s p t-5$ led to phenotypes perfectly reminiscent of $s m k-1$ loss, namely impaired longevity and resistance to oxidative stress but not to heat stress in daf-2 mutant animals, while spt-5 RNAi had no effect in wild type or in daf-2; daf-16 animals (Fig. 6c-e). Western blotting revealed that our knockdown eliminated $92 \%$ of the SPT-5 protein (Supplementary Fig. 9a, b), which indicated that as little as $\sim 8 \%$ of the physiological amount of SPT- 5 is sufficient to assure a basic level of genome-wide transcription but is insufficient to help PP4 $4^{\mathrm{SMK}-1}$ promote the induction of DAF-16 target genes and therefore the mechanisms described in this study.

Overall, this data is consistent with SPT- 5 being a key substrate by which PP4 4 MK-1 influences Pol II at many DAF-16 target genes and thereby fulfills its lifespan and stress resistance regulatory roles under low IIS.

SPT-5 is a direct substrate of PP4 $4^{\text {SMK-1 }}$. Having identified SPT-5, we wanted to test if it is a direct or rather an indirect substrate of $\mathrm{PP} 4^{\mathrm{SMK}-1}$. To address this, we conducted in vitro phosphatase assays. First, we immunoprecipitated the PP4SMK-1 complex from $C$. elegans and transferred it to the appropriate assay buffer. Next, we incubated the complex with a variety of different synthetically made phosphopeptides which resembled

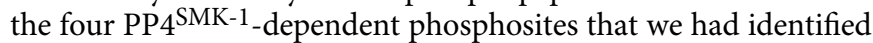
by our phosphoproteomics. As a negative control, we incubated the complex with a phosphopeptide that is known to be targeted by the closely related phosphatase $\mathrm{PP} 2 \mathrm{~A}^{29}$. Remarkably, we found that $\mathrm{PP} 4{ }^{\mathrm{SMK}-1}$ selectively dephosphorylated two of these phosphosites in SPT-5, S677, and S882, while it did not dephosphorylate the other phosphosites nor the negative control peptide (Supplementary Fig. 9c). To further confirm that this dephosphorylation was truly caused by $\mathrm{PP} 4^{\mathrm{SMK}-1}$ and not other C. elegans proteins or contaminants that might co-purify with the complex, we expressed a minimal PP4 ${ }^{\mathrm{SMK}-1}$ complex comprised of C. elegans PPH-4.1, PPFR-2, and SMK-1 recombinantly in S. cerevisiae. We purified this complex (see also Supplementary Fig. 9d), transferred it to assay buffer and conducted the same in vitro phosphatase assay as previously. Similar to the PP4SMK-1 complex purified from $C$. elegans, the recombinantly expressed minimal PP4 ${ }^{\mathrm{SMK}-1}$ complex also selectively dephosphorylated the sites S677 and S882 (Supplementary Fig. 9e). Finally, we tried to explore the purpose of the regulatory subunits, including SMK-1, in the $\mathrm{PP} 4^{\mathrm{SMK}-1}$ complex. Other studies have shown that regulatory subunits of PP4 can confer substrate binding and specificity ${ }^{10,11,30,31}$. Thus, we repeated our in vitro phosphatase assay one more time, now using only the catalytic subunit of PP4 ${ }^{\mathrm{SMK}-1}$, PPH-4.1, which we recombinantly expressed in E. coli. Notably, even though PPH-4.1 was sufficient to dephosphorylate the $\mathrm{PP} 4^{\mathrm{SMK}-1}$-targeted sites in vitro, the enzyme had now lost its selectivity and also dephosphorylated the SPT-5 phosphosite S904 or the PP2A target peptide with equal or even better efficiency (Supplementary Fig. 9f).

We conclude that PP4 $4^{\mathrm{SMK}-1}$ selectively dephosphorylates at least two phosphorylation sites in SPT-5 in vitro, which provides strong support for SPT-5 being a direct and specific substrate of $\mathrm{PP} 4^{\mathrm{SMK}-1}$ also in vivo.

\section{Discussion}

The transcription factor DAF-16/FOXO is one of the most central and powerful aging regulators across metazoans, relaying many distress signals into compensatory, aging-preventive transcriptional responses. Nevertheless, it is not self-sufficient but depends on other proteins' assistance. SMK-1, the first protein of this kind to be discovered ${ }^{8}$, is essential for DAF-16 to promote longevity and the resistance to many stresses under low IIS. This makes SMK-1 an important aging regulator in itself. Despite this prominence and the thorough genetic exploration of its functions ${ }^{8}$, it had remained unknown how SMK-1 is acting mechanistically. We could show that SMK-1 functions as part and through the catalytic activity of a specific Protein Phosphatase 4 complex, PP4 ${ }^{\text {SMK-1 }}$, and identified a direct substrate of this complex, SPT5 , whose dephosphorylation appears important for promoting DAF-16 functions under low IIS.

But how does $\mathrm{PP} 4^{\mathrm{SMK}-1}$-driven dephosphorylation of SPT-5 promote target gene expression by DAF-16? The literature describes SPT-5 predominantly as a transcriptional elongation factor and part of the conserved DSIF complex which it forms together with SPT $-4^{32}$. This complex binds to Pol II during transcriptional initiation and remains associated with it throughout transcription ${ }^{33,34}$. DSIF is important for Pol II's promoter-proximal pausing and transcriptional elongation ${ }^{35}$; but specifically SPT-5 was found to influence also other stages of transcription. Under certain circumstances it can influence Pol II's binding to promoter regions and transcriptional initiation ${ }^{36}$. This has been proposed to originate from elongation-engaged SPT-5 inferring epigenetic changes in promoter regions that 
facilitate new rounds of preinitiation complex (PIC) formation and thereby Pol II recruitment ${ }^{36}$. Furthermore, SPT- 5 facilitates promoter clearance by outcompeting PIC components like TFIIE from association with Pol $\mathrm{II}^{36,37}$. And last but not least, SPT-5 can influence transcriptional termination ${ }^{34}$. Several of these functions depend on the phosphorylation status SPT5 $5^{32,34}$. Upon transcriptional initiation, Pol II and SPT-5 await elongationpromoting signals by the kinase $\mathrm{P}-\mathrm{TEFb}$, which usually phosphorylates both proteins at "proline-directed" sites (S/T-P) in their CTDs and thereby drives pause site release and transcriptional elongation ${ }^{38-40}$. SPT-5's CTD remains phosphorylated throughout elongation and is dephosphorylated again near the transcriptional end site (TES), which contributes to transcriptional termination and release of Pol II and the DSIF complex from $\mathrm{DNA}^{34}$. In yeast, this dephosphorylation is conferred by Protein Phosphatase 1 (PP1) $)^{34}$.

All this prior work has already shown that SPT-5, even though it is mostly known as an elongation factor, can in principle influence most stages of transcription and that the phosphorylation state of SPT-5 is important for these roles. Such is consistent with our study showing that dephosphorylation of SPT-5 by $\mathrm{PP} 4{ }^{\mathrm{SMK}-1}$ promotes transcription initiating events at DAF-16 target genes under low IIS. Only how this works exactly remains an open question. The actual phosphorylation status of the

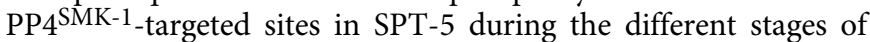
transcription as well as off the DNA remains unknown. We can only say that they are overall hyperphosphorylated upon $s m k-1$ RNAi and that none of these sites, even though some of them are located within SPT-5's CTD, are the canonical P-TEFb target sites mentioned above. Thus, we also do not know if PP4 $4^{\text {SMK-1 }}$ directly counteracts P-TEFb or whether it may dephosphorylate sites targeted by other kinases. The exact role of all phosphorylation sites on SPT- 5 both inside and outside of its CTD, their turnover in the course of transcription, and the full portfolio of kinases and phosphatases that target them will be interesting topics of future investigation.

We would like to note that $\mathrm{PP} 4{ }^{\mathrm{SMK}-1}$ predominantly promotes the step of Pol II recruitment to promoter regions, which is an event when SPT-5 has not yet bound to Pol II. Thus, in this context the effect of SPT- 5 on Pol II should be indirect, which by analogy to previous observations ${ }^{36}$ may be caused by elongationengaged SPT- 5 conferring epigenetic changes in promoter regions that facilitate Pol II recruitment.

In a last effort to supplement our understanding of the

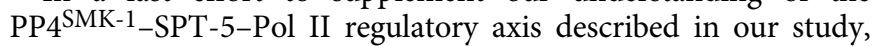
we addressed three remaining questions. First, is it possible that PP4 4 MK-1 impacts transcription initiating events by regulating SPT-5's recruitment to promoter/TSS regions? We addressed this by ChIP-seq analysis of DAF-16/SMK-1 co-activated genes in daf-2 animals and found that knockdown of $s m k-1$ had no significant effect on SPT-5 recruitment (Supplementary Fig. 10a). This suggests that it is not absence of SPT-5 but its altered phosphorylation status that impairs transcription initiating events in the absence of PP4 $4^{\mathrm{SMK}-1}$. Second, we asked if, analogous to PP1 in yeast, $\mathrm{PP} 4{ }^{\mathrm{SMK}-1}$ also influences transcriptional termination. Again through ChIP-seq in daf-2 animals, we observed that smk-1 RNAi causes a moderate shift of SPT-5 and Pol II distribution into the downstream direction, specifically at TESs of DAF-16/ SMK-1-co-activated genes (Supplementary Fig. 10b, c). This result would be consistent with also transcriptional termination being moderately delayed at these genes. However, it remains to be tested whether and to what extend such termination defects would actually contribute to reduced expression of these genes. Third, we asked where PP4 ${ }^{\mathrm{SMK}-1}$ encounters SPT-5 and dephosphorylates it. We conducted ChIP-seq of SMK-1 using SMK-1:: GFP-expressing daf-2 mutant C. elegans. Association of SMK-1 with chromatin was extremely weak. However when looking cumulatively at all genes genome-wide, we observed a profile that would be consistent with SMK-1 binding to SPT-5 specifically during transcriptional elongation and dissociating just before the TES is reached (Supplementary Fig. 10d). This data argues that dephosphorylation of SPT- 5 by PP $4^{\text {SMK- } 1}$ may occur during transcriptional elongation prior to termination. However, we cannot exclude that $\mathrm{PP} 4^{\mathrm{SMK}-1}$ acts also in the nucleoplasm, where it would dephosphorylate SPT-5 to prepare it for engagement in new rounds of transcription.

A remarkable aspect of our study is that PP4 $4^{\mathrm{SMK}-1}$ influences transcription through a highly conserved and common component of the transcription regulatory machinery, namely SPT-5. Nevertheless, it does not affect transcription genome-wide but only of a limited gene set. Such specificity actually has precedence. Studies in zebrafish and flies have similarly shown that mutations of SPT-5 affect only parts of the transcriptome $e^{41,42}$. But where would this specificity come from? We could see in Fig. $5 c$ that promoter regions of DAF-16/SMK-1-co-activated genes may have unique properties, resulting in Pol II clearing these promoter regions more slowly. We propose that this makes them more sensitive to defects in transcription initiating events. Looking for sequence features that may distinguish these promoter regions, we found them enriched for TATA-boxes (TATA) $\left(p=2.45 \times 10^{-4}\right.$; Supplementary Table 8$)$, while initiator elements (Inr) were not enriched (Supplementary Table 8). In contrast, DAF-16-activated promoters that do not depend on SMK-1 for their activation are depleted of both TATA and Inr (Supplementary Table 8). Notably, presence of TATA-boxes has been associated with genes for which transcriptional initiation is particularly rate limiting ${ }^{43}$. Considering all these observations, it would be consistent that loss of PP4 $4^{\mathrm{SMK}-1}$ and the resulting impairment of transcription initiating events selectively impair the expression of this subset of DAF-16 target genes. Regarding the purpose of such regulatory mechanism we can only speculate. It may be a feature of genes that need the ability to be rapidly and/or robustly induced in response to certain stimuli, i.e., a drop in IIS. Consistent with such notion, a recent study in humans showed particular involvement of SPT-5 in regulating the transcriptional initiation of rapidly-induced immune response genes $^{36}$. Nevertheless, future studies will have to explore this in more detail.

It is also notable that $\mathrm{PP} 4^{\mathrm{SMK}-1}$, although it is required for most DAF-16 functions under low IIS, is irrelevant for some, e.g., for promoting resistance to heat stress (Fig. 3). Consistently, we observed that depletion of SPT-5 impaired longevity and oxidative stress resistance but not heat stress resistance under low IIS (Fig. 6c-e). This indicates that some DAF-16 target genes are regulated in a fundamentally different manner that does not involve the PP4 ${ }^{\mathrm{SMK}-1}-\mathrm{SPT}-5-\mathrm{Pol}$ II axis, e.g., due to different promoter characteristics or DAF-16 fulfilling these functions in synergy with other transcription factors that impose different mechanisms of transcriptional control at these loci. Intriguingly, for heat shock response genes it is already known that DAF-16 regulates them combinatorially together with HSF-1 ${ }^{44}$; and it has been shown that expression of HSF-1 target genes is not controlled on the level of Pol II recruitment or promoter clearance but rather by pause site release ${ }^{45}$.

Taken together, our study provides substantial mechanistic insight and suggests a possible model for how SMK-1 promotes the expression of many DAF-16-activated genes under low IIS. Figure 7 illustrates this model and highlights the transcriptional events that seem affected when $\mathrm{PP} 4^{\mathrm{SMK}-1}$ activity is missing.

Intriguingly, SMK-1 may influence not only the expression of DAF-16-dependent genes but also the targets of other transcription factors, and it may promote longevity also in conditions 
a
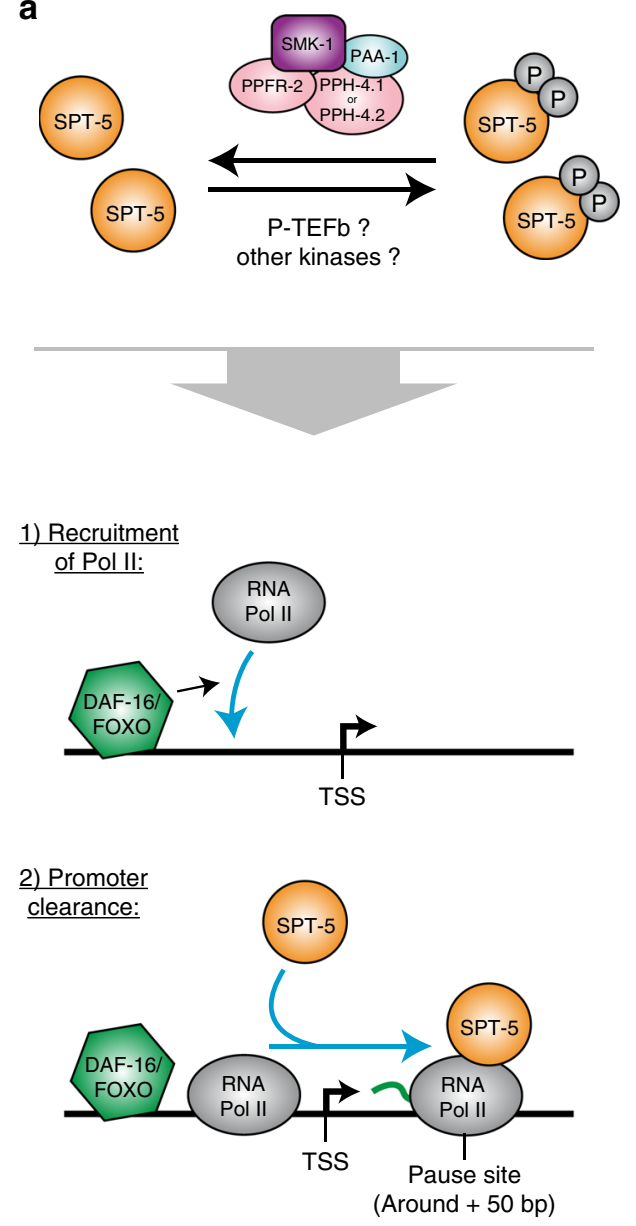

3) Transcriptional elongation:

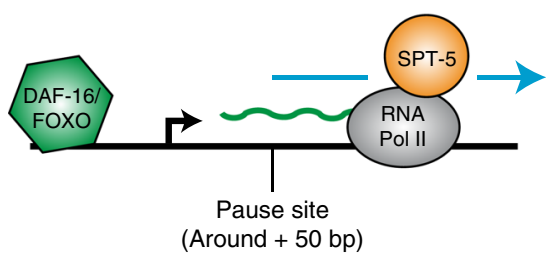

4) Transcriptional termination:

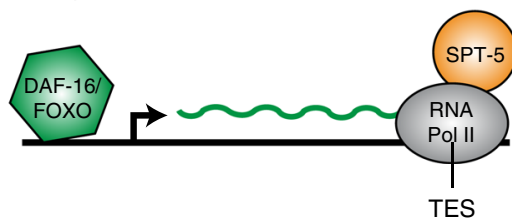

(P)

Phosphorylation sites targeted by $\mathrm{PP} 4^{\text {SMK-1 }}$

besides low IIS. We obtained preliminary support for this notion when we compared the genes regulated by SMK-1 under low IIS to the genes regulated by two other transcription factors that also contribute to the longevity of daf-2 mutant $C$. elegans $-\mathrm{SKN}-1^{46}$ and HLH-30 ${ }^{18}$. In particular, genes regulated by HLH-30 were strongly dependent on SMK-1 for their regulation (Supplementary Table 9). A previous study investigating dietarily restricted $C$. elegans (eat-2 mutants) showed that their longevity, which b
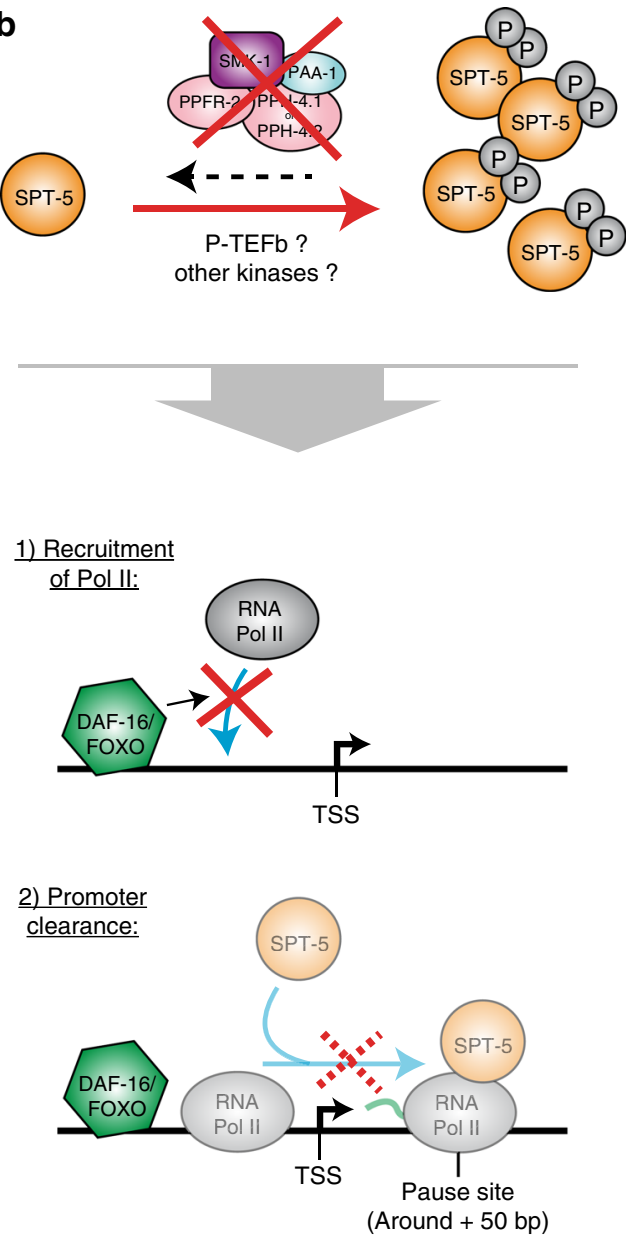

3) Transcriptional elongation:

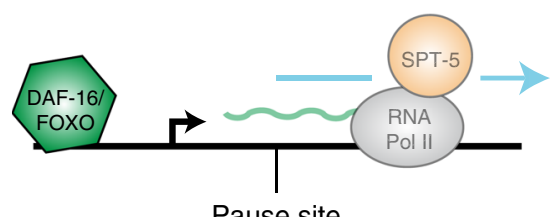

Pause site

(Around +50 bp)

4) Transcriptional termination:

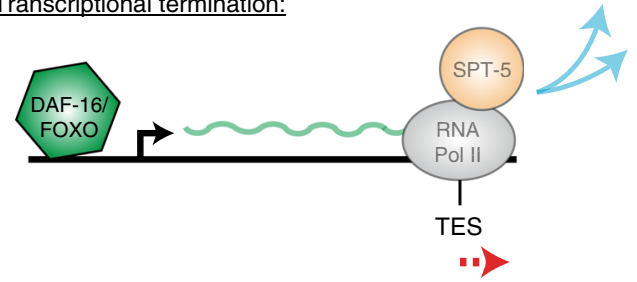

$X: \cdots, \rightarrow \cdots$ Defects caused by loss of PP4

depends not on DAF-16 but rather PHA-4, was also dependent on SMK- ${ }^{47}$. Finally, it was shown that SMK-1 is essential for the increased lifespan of mitochondrial electron transport chain mutants ${ }^{8}$. This points to an involvement of $\mathrm{PP} 4^{\mathrm{SMK}-1}$ also with other aging-regulatory signaling pathways and their downstream transcription factors, which will be interesting to explore further.

Finally we would like to highlight the strong conservation of all components mentioned in our study across metazoans. Two 
Fig. 7 Model illustrating the roles of PP4 ${ }^{\text {SMK-1 }}$ and SPT-5 for the transcription of DAF-16/FOXO target genes. SPT-5 is known to get phosphorylated at various sites. Some of these sites, in particular proline-directed sites in the SPT-5 CTD, are targeted by the kinase P-TEFb, to promote transcriptional elongation. However, our study shows that other phosphorylation events in- and outside the SPT-5 CTD occur, too. We do not know the kinases which phosphorylate these other sites, be it P-TEFb or rather distinct kinases. However, we found that several of these other sites are dephosphorylated by the phosphatase PP4 ${ }^{\mathrm{SMK}-1}$. a Normally, the phosphorylation state of these PP4 ${ }^{\mathrm{SMK}-1}$-targeted sites is in balance. We unfortunately do not know during which stages of transcription they are phosphorylated nor when their dephosphorylation by PP4 ${ }^{\mathrm{SMK}-1}$ has to occur; but ultimately this balance allows for the efficient expression of DAF-16 target genes under low IIS. $\mathbf{b}$ In contrast, when PP4SMK-1 activity is missing, this balance shifts toward an overabundance of SPT-5 that is phosphorylated at these sites. Even though we do not understand the full mechanistic basis to it, this shift toward increased SPT-5 phosphorylation interferes with Pol II recruitment to promoter regions and may also mildly affect promoter clearance, leading to impaired transcription initiating events. Finally, a mild delay in transcriptional termination may occur, too. The red crosses and arrow indicate these phenotypes.

previous studies in $S$. cerevisae have observed a physical interaction between Psy2 (the S. cerevisiae ortholog of SMK-1) and Spt5 in tandem affinity purification-MS/MS experiments ${ }^{9}$, and we were able to confirm the existence of a PP4SMEK_SUPT5H complex under low IIS conditions in human cells (Supplementary Fig. 8c). This suggests that a PP4 $4^{\text {SMEK_SUPT5H-Pol II axis }}$ influencing the expression of FOXO target genes might exist in humans, too. It will be important to verify its conservation and whether it likewise promotes stress resistance and longevity.

\section{Methods}

C. elegans strains and alleles. For a complete list of strains used in this study, see Supplementary Table 1 . Alleles obtained from other labs were 4 times outcrossed to the lab's N2 background. All C. elegans strains were grown and maintained on Escherichia coli OP50 using standard methods ${ }^{48}$.

RNAi by feeding. C. elegans were grown on the E. coli strain HT115 containing dsRNA-expressing plasmids targeting the genes of interest. RNAi clones either were obtained from published collections ${ }^{49,50}$ or were newly made. RNAi clones for daf-2, $p p h-4.1, p p h-4.2, p p f r-2, s p t-4$, and $e m b-5$ were used from ${ }^{49}$ and RNAi clones for $d a f-16, s p t-5$, and $s m k-1$ were used from ${ }^{50}$. For screening purposes, all available RNAi clones from ${ }^{49,50}$ that targeted the genes of interest were used. RNAi clones targeting $p p f r-1$ and $p p f r-4$ were newly made by PCR-amplifying approximately 1 $\mathrm{kb}$ of cDNA or gDNA sequence located in the coding region of the gene of interest, cloning it into L4440 by AT-ligation, and then transforming the resulting construct into E. coli HT115. PCR primers CAACAACTTGGCGGTGCTATC (forward) and GTTCCGGATTGCAAGCAACG (reverse) were used on gDNA to make the $p p f r-1$ RNAi clone; and CAAGCGCTTTACGATCCGAG (forward) and CATC-

CACGTCGATGATCATCC (reverse) were used on cDNA to make the $p p f r-4$ RNAi clone. E. coli HT115 containing the empty plasmid L4440 served as negative control in the RNAi experiments. Combined knockdown of either the two PP4 SMK${ }^{1}$ catalytic subunits $p p h-4-1$ and $p p h-4.2$ or of $p p h-4.2$ and $s m k-1$ was achieved by a 1:1 mixture of their RNAi bacteria.

Unless stated otherwise, RNAi feeding was initiated at the L1 stage. In most RNAi experiments, a RNAi-sensitized strain background (eri-1(mg366)) was used, to yield better knockdown efficiency ${ }^{51}$. Usage of this background is always stated in the respective figure legends.

Generating transgenic $\boldsymbol{C}$. elegans. To generate $\mathrm{PPH}-4.1:$ GFP-expressing C. elegans, the promoter of $p p h-4.1$ (the first $600 \mathrm{bp}$ upstream of the START codon) was cloned from genomic DNA and the spliced $p p h-4.1$ coding region from cDNA of $\mathrm{N} 2$ animals. These sequences were then fused by PCR and cloned into the GFPfusion expression vector pPD95_75 (Addgene), to yield the desired $p p h-4.1 P:: p p h$ 4.1::gfp construct. Next, we coinjected this plasmid $(30 \mathrm{ng} / \mu \mathrm{l})$ with ScaI-digested pUC19 $(40 \mathrm{ng} / \mu \mathrm{l})$ and EcoRI-digested pRF4 $(30 \mathrm{ng} / \mu \mathrm{l})$ to yield complex arrays marked by rol-6(su1006). Injections were performed using an inverted DIC microscope (Zeiss) in combination with an InjectMan 4 and FemtoJet $4 \mathrm{i}$ (Eppendorf). Rollers were selected from the F1 progeny and lines were established.

C. elegans expressing catalytically dead PPH-4.1(R262L) were generated in the same way-only that we mutated the $p p h-4.1 P:: p p h-4.1:: g f p$ construct by sitedirected mutagenesis, replacing AGA by CTT.

Microscopy and nuclear translocation assays. For imaging, L4 stage worms were paralyzed by 2,3-butanedione monoxime (BDM), mounted on $2 \%$ agarose pads, and imaged using a Zeiss Axio Observer $\mathrm{Z} 1$ microscope at $20 \mathrm{x}$ or $40 \mathrm{x}$ magnifications.

To investigate the effect of SMK-1 on nuclear entry of DAF-16, daf-2(e1370) mutant animals expressing DAF-16::GFP were grown from the $\mathrm{L} 1$ stage at $15^{\circ} \mathrm{C}$ on either control or $s m k-1$ RNAi. On day 2 of adulthood, animals were shifted to $25^{\circ} \mathrm{C}$, upon which moment nuclear entry was scored in the intestine of the animals. To investigate the effect of SMK-1 on nuclear exit of DAF-16, animals were grown in the same way to day 1 adulthood, shifted to $25^{\circ} \mathrm{C}$ for $24 \mathrm{~h}$ to reach full nuclear entry of DAF-16, and then shifted back to $15^{\circ} \mathrm{C}$, upon which moment nuclear exit was scored in the intestine of the animals. Scoring was done blinded, using a Zeiss Axio Zoom V16 microscope.

Testing the knockdown efficiency of spt-5 RNAi. daf-2(e1370) animals expressing SPT-5::GFP were synchronized by bleaching, grown on HT115 bacteria without dsRNA-expressing plasmids at $15^{\circ} \mathrm{C}$ until the $\mathrm{L} 4$ stage, washed in antibiotics to remove the HT115, and transferred to plates seeded with either control or spt-5 RNAi bacteria. 5-fluoro- 2 '-deoxyuridine (FUDR) was added to a final concentration of $50 \mu \mathrm{M}$ to prevent progeny production and the plates were shifted to $25^{\circ} \mathrm{C}$. On day 2 of adulthood, 50 worms per condition were harvested and analyzed by SDS-PAGE and western blotting. For detection of SPT-5::GFP we used monoclonal mouse anti-GFP antibody (Roche) and for detection of actin we used monoclonal (C4) mouse anti-actin antibody-both diluted 1:1000 in TBST containing $5 \%$ milk powder. For quantification, band intensities were determined using the Fiji image processing package (ImageJ) and data were normalized to actin. This experiment was repeated 3 times. Unpaired $t$ test (two-tailed) was used for comparisons and statistical analysis.

Lifespan assays. C. elegans were synchronized by bleaching and grown, depending on the experiment, on either OP50 or RNAi bacteria at $15^{\circ} \mathrm{C}$ until the late $\mathrm{L} 4$ stage. In cases where the RNAi bacteria would lead to developmental arrest, animals were initially grown on $\mathrm{HT} 115$ bacteria without dsRNA-expressing plasmids at $15^{\circ} \mathrm{C}$ and only at the L4 stage washed in antibiotics to remove the HT115 and transferred to RNAi bacteria. In all cases 5-fluoro-2'-deoxyuridine (FUDR) was added to a final concentration of $50 \mu \mathrm{M}$ at the late $\mathrm{L} 4$ stage to prevent progeny production and the plates were shifted to $25^{\circ} \mathrm{C}$. Survival of animals was determined every $2-3$ days by scoring movement upon prodding with a platinum wire ${ }^{52}$.

Stress survival assays. C. elegans were synchronized by bleaching and grown, depending on the experiment, on either OP50 or RNAi bacteria at $15^{\circ} \mathrm{C}$ until the late $\mathrm{L} 4$ stage. In cases where the RNAi bacteria would lead to developmental arrest, animals were initially grown on HT115 bacteria without dsRNA-expressing plasmids at $15^{\circ} \mathrm{C}$ and only at the L4 stage washed in antibiotics to remove the HT115 and transferred to RNAi bacteria. In all cases FUDR $(50 \mu \mathrm{M}$ final concentration) was added at the late L4 stage to prevent progeny production and the plates were shifted to $25^{\circ} \mathrm{C}$. At day 2 of adulthood, animals were transferred to plates containing $6 \mathrm{mM}$ tBOOH (for oxidative stress assays), the temperature was shifted to $32{ }^{\circ} \mathrm{C}$ (for heat stress assays), or animals were exposed to $1500 \mathrm{~J} / \mathrm{m}^{2} \mathrm{UV}$ light (for UV stress assays). Their survival was recorded and analyzed by a fully automated lifespan machine ${ }^{16}$.

Immunoprecipitation from C. elegans. Growth of $C$. elegans to large scale, harvesting, lysis, IP of the bait proteins, and eventual analysis of the precipitated material by tandem mass spectrometry were conducted as previously described ${ }^{17}$ At first, the animals were grown asynchronously at $15^{\circ} \mathrm{C}$ to scale, then shifted for $16 \mathrm{~h}$ to $25^{\circ} \mathrm{C}$ and harvested. Animals were transferred to lysis buffer $(50 \mathrm{mM}$ HEPES at pH 7.4, $1 \mathrm{mM}$ EGTA, $1 \mathrm{mM} \mathrm{MgCl} 2,150 \mathrm{mM} \mathrm{KCl,} \mathrm{10 \%} \mathrm{(v/v)} \mathrm{glycerol,}$ Complete without EDTA (Roche), $1 \mathrm{mM}$ phenylmethyl sulphonyl fluoride, and phosphatase inhibitors (Sigma)) and lysed by grinding under liquid nitrogen. NP40 was added to $0.05 \%(\mathrm{v} / \mathrm{v})$, and the resulting lysate was cleared at $20,000 \mathrm{~g}$. mCherry-tagged bait proteins were immunoprecipitated using a rabbit-antimCherry antibody that we generated in the lab and coupled to Protein A resin (Biorad). GFP-tagged bait proteins were immunoprecipitated using GFP-trap resin (Chromotek). Immunoprecipitated proteins were eluted using $100 \mathrm{mM}$ glycine at $\mathrm{pH}$ 2.6. As negative controls for background subtraction, the same purifications and later mass spectrometry analyses were conducted using wild type (untagged) $C$. elegans.

Mass spectrometry to identify co-purifying proteins. Immunoprecipitated proteins were digested overnight at $\mathrm{pH} 8.3$ using sequencing-grade trypsin. Resulting peptides were separated by microcapillary reverse-phase chromatography 
and identified by online tandem mass spectrometry using an LTQ Orbitrap XL hybrid linear ion trap-orbitrap high-resolution mass spectrometer (Thermo). Tandem mass spectra produced by collision induced dissociation (CID) in the ion trap were searched against the Wormpep database (version 246) using ProLuCID ${ }^{53}$ and DTASelect ${ }^{54}$. Enzyme specificity was set to trypsin, allowing cleavage Nterminally to proline. Further modifications were cysteine carbamidomethylation (fixed), as well as methionine oxidation and phosphorylation of the amino acids $\mathrm{S}$, $\mathrm{T}$, or Y (all variable). False discovery rates (FDRs) were determined by searching the mass spectra against the reversed sequences of the Wormpep database. Proteins had to be identified by at least two independent peptides, resulting in an FDR below $1 \%$. Finally, samples were compared using Contrast ${ }^{54}$. Any proteins that were identified also in the control purifications (from untagged C. elegans lysates) were likely contaminants and thus eliminated from Fig. $1 \mathrm{~b}$ and Supplementary Tables 2, 3 .

Co-immunoprecipitation from HEK293T cells. The HEK293T cells (ATCC, tested to be void of mycoplasma contamination) were grown in advanced Dulbecco's modified Eagle's medium/F12 supplemented with 10\% fetal bovine serum and then transfected in $9 \mathrm{~cm}$ dishes with $15 \mu \mathrm{g}$ of plasmid DNA for GFP-SMEK1 (Addgene), using the Profection mammalian transfection system (Promega). $12 \mathrm{~h}$ post transfection, medium was replaced with fresh antibiotic-free media and LY294002 was added at a final concentration of $20 \mu \mathrm{M}$. After $48 \mathrm{~h}$ of LY294002 treatment, cells were harvested by scraping and frozen in liquid nitrogen. For the co-IPs, these cell pellets were thawed into lysis buffer (50 mM HEPES at pH 7.4, 1 mM EGTA, $1 \mathrm{mM} \mathrm{MgCl}$, $150 \mathrm{mM} \mathrm{KCl}, 10 \%$ (v/v) glycerol, Complete (Roche), 1 $\mathrm{mM}$ phenylmethyl sulphonyl fluoride, phosphatase inhibitors (Roche), and $0.5 \%$ $(\mathrm{v} / \mathrm{v}) \mathrm{NP}-40)$ and lysed by shearing through a G30 syringe. Lysates were cleared at 20,000 g. GFP-tagged proteins were immunoprecipitated using GFP-Trap resin (Chromotek) and eluted by boiling in $2 \times$ sample buffer. Input (IN) and eluate (IP) samples were analyzed by SDS-PAGE and western blotting.

Production of recombinant proteins in E. coli. The cDNA of $p p h-4.1$ was cloned into the GST-fusion expression vector pGEX-4T1 and the resulting plasmid transformed into BL21(DE3)-RIPL (Agilent). The resulting bacterial strain was grown to an $\mathrm{OD}_{600}$ of 0.6 at $37^{\circ} \mathrm{C}$ and $230 \mathrm{rpm}$ and then induced with IPTG for 18 $\mathrm{h}$ at $18^{\circ} \mathrm{C}$ and $230 \mathrm{rpm}$. Next the bacteria were harvested and frozen in liquid nitrogen. For the purification of PPH-4.1, the bacteria were thawed into TBS buffer containing $1 \mathrm{mM}$ EDTA, $1 \mathrm{mM}$ DTT, and Complete without EDTA (Roche), and incubated with lysozyme for $30 \mathrm{~min}$ at $4{ }^{\circ} \mathrm{C}$. Resulting lysate was sonicated, Triton X-100, RNase A (Roche), and DNase I (Invitrogen) were added to concentrations of $1 \%(\mathrm{v} / \mathrm{v}), 10 \mu \mathrm{g} \mathrm{ml}^{-1}$, and $5 \mu \mathrm{g} \mathrm{ml}^{-1}$, respectively, and the lysate then incubated for another $30 \mathrm{~min}$ at $4{ }^{\circ} \mathrm{C}$. Glutathione-Sepharose $4 \mathrm{~B}$ resin (GE Healthcare) was added to the lysate and incubated rotating for $2 \mathrm{~h}$ at $4{ }^{\circ} \mathrm{C}$. Finally, the resin was washed in TBS, resuspended in TBS, and then used for in vitro phosphatase assays.

Production of recombinant proteins in S. cerevisiae. The cDNA of $p p h-4.1$ was fused to a TAP tag and then used to replace the cdt1 gene in the plasmid pJF255, creating a Gall promoter-driven expression construct. The cDNA of ppfr-2 was used to replace $\mathrm{mcm} 7$ and the cDNA of $s m k-1$ was fused to a $\mathrm{HA}_{3}$ tag and then used to replace $\mathrm{mcm} 6$ in the plasmid $\mathrm{pJF} 4^{55}$, creating a Gall-10 promoter-driven expression construct. Both expression constructs were integrated into the genome of the S. cerevisiae strain CB3245 (MATa, pep4A::HPH). Resulting cells were grown at $30{ }^{\circ} \mathrm{C}$ and $200 \mathrm{rpm}$ in YEP medium containing $2 \%$ raffinose to an $\mathrm{OD}_{600}$ of 1.0 , then $2 \%$ galactose was added to induce the overexpression and the cells were grown for an additional $4 \mathrm{~h}$ at $30^{\circ} \mathrm{C}$ and $200 \mathrm{rpm}$. Cells were harvested by centrifugation, washed with IPP150 buffer (50 mM Tris- $\mathrm{HCl}(\mathrm{pH} 8.0), 150 \mathrm{mM} \mathrm{NaCl}, 10 \%$ glycerol, $0.1 \% \mathrm{NP}-40$, and $1 \mathrm{mM}$ dithiothreitol (DTT)) and ground in liquid nitrogen using a SPEX 6870 freezer mill. Ground cells were dissolved in $1 \mathrm{ml}$ IPP150 buffer containing Complete without EDTA (Roche), $10 \mathrm{mM} \mathrm{MgCl}_{2}$ and $12.5 \mathrm{U}$ Benzonase (Sigma), and incubated for $1 \mathrm{~h}$ at $4{ }^{\circ} \mathrm{C}$. The lysate was cleared by centrifugation for $20 \mathrm{~min}$ at $16.000 \mathrm{~g}$, and the purification of the TAP-tagged protein was performed as described before ${ }^{56}$ with slight modifications. As beads for the purification we used IgG Sepharose 6 Fast Flow (GE Healthcare). Glutathione-Sepharose 4B beads (GE Healthcare) were used for the mock purification. Beads were divided into two tubes and washed with GF150 buffer (20 mM HEPES (pH 7.5), $150 \mathrm{mM} \mathrm{NaCl}, 10 \%$ glycerol, $0.1 \%$ NP-40, and $1 \mathrm{mM}$ DTT). Finally, the PPH-4.1::TAP was eluted with TEV protease and used for in vitro phosphatase assays.

For detection of PPH-4.1::TAP by western blotting we used anti-TAP antibody (antibodies online.com, Cat\#ABIN398491) diluted 1:1000 in TBST containing 2\% $(\mathrm{w} / \mathrm{v})$ milk powder (Sigma). For detection of SMK-1::HA antibody (Abcam, cat \# ab9110) diluted 1:3000 in TBST containing 5\% (w/v) milk powder.

PP4 ${ }^{\text {SMK-1 }}$ purification from C. elegans for in vitro assays. daf-2(e1370) animals expressing SMK-1::GFP were grown asynchronously to scale at $15^{\circ} \mathrm{C}$, then shifted for $16 \mathrm{~h}$ to $25^{\circ} \mathrm{C}$ and harvested. Animals were transferred to lysis buffer $(50 \mathrm{mM}$ HEPES at pH 7.4, $1 \mathrm{mM}$ EGTA, $1 \mathrm{mM} \mathrm{MgCl}, 150 \mathrm{mM} \mathrm{KCl}, 10 \%$ (v/v) glycerol, Complete without EDTA (Roche)), and lysed by grinding under liquid nitrogen. NP-40 was added to $0.05 \%(\mathrm{v} / \mathrm{v})$, and the resulting lysate was cleared at $20,000 \mathrm{~g}$.
The SMK-1::GFP was immunoprecipitated using GFP-trap resin (Chromotek), the resin washed, and then resuspended in IP-EL buffer $(50 \mathrm{mM}$ HEPES at $\mathrm{pH} 7.4,1$ mM EGTA, $1 \mathrm{mM} \mathrm{MgCl} 2,150 \mathrm{mM} \mathrm{KCl}$, and $10 \%$ (v/v) glycerol), to be used in the in vitro phosphatase assays.

In vitro phosphatase assay. We designed phosphopeptides for each $\mathrm{PP} 4^{\mathrm{SMK}-1}$ dependent phosphosite on SPT-5 (S671: PMVSRMAS(p)PNPMAS, S677: ASPNPMAS(p)PRHSSGG, S882: TPAYGS(p)ADGAR, S904: TPAYGS(p)TEGGR). In addition, we included a known PP2A substrate peptide ("PP2A target"), derived from the retinoblastoma protein $\mathrm{RB}^{29}$ (PP2A target: INGSPRT(p)PRRGQNR), as a negative control. These peptides were synthesized (95\% purity, TAG Copenhagen $\mathrm{A} / \mathrm{S}$ ) and, prior to assays, freshly diluted to $1 \mathrm{mM}$ final concentration in pNPP Ser/ Thr Assay Buffer (50 mM Tris- $\mathrm{HCl}(\mathrm{pH} 7.0), 100 \mu \mathrm{M} \mathrm{CaCl} 2$ ). Purified material (either $10 \mu \mathrm{l}$ beads or $10 \mu \mathrm{l}$ eluted protein) was mixed with $40 \mu \mathrm{l}$ of $1 \mathrm{mM}$ Phosphopeptide solution. Samples were incubated at RT for $15 \mathrm{~min}$ on a shaking incubator at $800 \mathrm{rpm}$. All these assays were conducted in triplicate. For the eventual detection of the released phosphate, samples were transferred to 96-well half area assay plates (Corning) and diluted fivefold in pNPP Ser/Thr Assay Buffer to reach detectable range. We used a Malachite Green Phosphate Assay Kit (Sigma, cat \# MAK307) and performed the assay according to the manufacturer's instructions. One-way ANOVA and post hoc Dunnett's test were used for statistical calculations and multiple comparisons of each sample with the control substrate ("PP2A target").

Phos-tag SDS-PAGE analysis. Worms were grown on either control or smk-1 RNAi bacteria until day 2 of adulthood and then harvested into a lysis buffer ( 20 $\mathrm{mM}$ MOPS (pH 7.2), $15 \mathrm{mM} \mathrm{MgCl}_{2}, 100 \mathrm{mM} \mathrm{NaCl}, 1 \%$ (v/v) Triton X-100, $1 \mathrm{mM}$ DTT, Complete without EDTA (Roche), $1 \mathrm{mM}$ phenylmethyl sulphonyl fluoride, and PhosSTOP (Roche)). Samples were lysed using a FastPrep and silicon carbide beads with $1.0 \mathrm{~mm}$ diameter (BioSpec). Per condition, material equivalent to 50 worms was loaded onto SuperSep Phos-tag $(50 \mu \mathrm{M})$ 10\% precast gels (Wako) and then analyzed by SDS-PAGE and western blotting. Monoclonal mouse anti-GFP antibody (Roche) diluted 1:1000 in TBST containing 5\% milk powder was used to detect DAF-16::GFP in the different samples.

Sample preparation for phosphoproteomic analyses. daf-2(e1370) mutant worms were synchronized by bleaching, seeded as L1 larvae onto plates containing either control or $s m k-1$ RNAi bacteria, and grown at $15^{\circ} \mathrm{C}$ until the L4 stage. Plates were then shifted to $25^{\circ} \mathrm{C}$ and FUDR $(50 \mu \mathrm{M}$ final concentration) was added to prevent progeny production. On day 2 of adulthood, animals were harvested, washed, and frozen in liquid nitrogen. Animals were lysed by grinding under liquid nitrogen, using a cryomill (Retsch). Then $300 \mathrm{mg}$ of worm powder were thawed and $200 \mu \mathrm{l}$ of the resulting lysate transferred to a new tube, where it was diluted with $300 \mu$ of lysis buffer ( $8 \mathrm{M}$ Urea, $1 \%$ SDS, $50 \mathrm{mM}$ Tris $\mathrm{pH} 8.5$, protease inhibitors (Roche), and phosphatase inhibitors (Roche)). Samples were then sonicated on ice to shear the DNA and cleared by centrifugation for $10 \mathrm{~min}$ at $4{ }^{\circ} \mathrm{C}$ and $20,000 \mathrm{~g}$. Supernatants were transferred to new tubes and their protein concentration measured using BCA assay (Pierce). Six hundred micrograms of proteins were reduced by adding DTT to a final concentration of $5 \mathrm{mM}$ and incubating for $1 \mathrm{~h}$ at $25^{\circ} \mathrm{C}$. Samples were then alkylated by adding iodoacetamide to a final concentration of $15 \mathrm{mM}$ and incubating for $30 \mathrm{~min}$ in the dark at room temperature. Excess iodoacetamide was quenched by adding DTT to an additional 10 $\mathrm{mM}$ of final concentration. Proteins were isolated by methanol/chloroform precipitation, redissolved in $100 \mu \mathrm{l}$ of $50 \mathrm{mM}$ Tris $\mathrm{pH} 8.5$ containing $8 \mathrm{M}$ urea, and then diluted with an equal volume of $50 \mathrm{mM}$ Tris $\mathrm{pH}$ 8.5. Proteins were digested by adding $10 \mu \mathrm{g}$ of LysC (Promega) and incubating for $6 \mathrm{~h}$ at $24^{\circ} \mathrm{C}$ and $300 \mathrm{rpm}$. Next, three volumes of $50 \mathrm{mM}$ Tris $\mathrm{pH} 8.5$ were added to reach a final urea concentration of $1 \mathrm{M} .10 \mu \mathrm{g}$ of LC grade trypsin (Promega) was added and samples were incubated overnight at room temperature. After digestion, TFA was added to $0.5 \%(\mathrm{v} / \mathrm{v})$ and samples were centrifuged for $10 \mathrm{~min}$ at $4{ }^{\circ} \mathrm{C}$ and $20,000 \mathrm{~g}$. The pellet was discarded and the supernatant desalted using $\mathrm{C} 18$ SepPak cartridges (Waters). Phosphopeptides were enriched using a $\mathrm{TiO}_{2}$ Phosphopeptide Enrichment and Clean-up Kit (Pierce) according to the manufacturer's recommendations.

Mass spectrometry for phosphoproteomic analyses. Peptides were separated using a $50 \mathrm{~cm}$ EASY-Spray ${ }^{\mathrm{mm}}$ LC column (Thermo Scientific) and the EASY-LC 1000 chromatography system (Thermo Scientific). Separation was achieved by a $120 \mathrm{~min}$ linear gradient from 2 to $26 \%$ acetonitrile in $0.1 \%$ formic acid at a flow rate of $300 \mathrm{~nL} / \mathrm{min}$. Eluting peptides were ionized by electron spray and analyzed by an Orbitrap Fusion mass spectrometer (Thermo Fisher Scientific). The survey MS spectrum was acquired at a resolution of $R=120,000$ in the range of $\mathrm{m} / \mathrm{z}$ 200-2000. MS/MS data for the 20 most intense precursors were obtained using higher-energy collisional dissociation for ions with a charge $z>1$ at a resolution of $R=15,000$

Data analysis for phosphoproteomic analyses. RAW data from the mass spectrometer were analyzed using MaxQuant 1.5.3.3057. A FDR of 0.01 for proteins and peptides and a minimum peptide length of six amino acids were required. The Andromeda search engine ${ }^{58}$ was used to search the MS/MS spectra against the 
Uniprot C. elegans database (WBcel235) combined with 262 common contaminants and concatenated with the reversed versions of all sequences. Enzyme specificity was set to trypsin, allowing cleavage $\mathrm{N}$-terminally to proline. Further modifications were cysteine carbamidomethylation (fixed), as well as protein $\mathrm{N}$ terminal acetylation, asparagine and glutamine deamidation, methionine oxidation, and phosphorylation of the amino acids S,T, or Y (all variable). Further evaluation of the data provided by MaxQuant was performed using $\mathrm{R}^{59}$. Proteins' relative intensities were normalized to the total protein signal. Differences in relative protein abundances between treated and control samples were assessed by moderated $t$ test using the R-package limma ${ }^{60}$. Benjamini-Hochberg correction for multiple comparisons was used.

Transcriptional reporter assays. The sod-3p::GFP-expressing C. elegans strains CF1553 or AS23 were synchronized by bleaching and seeded as L1 larvae onto plates containing RNAi bacteria, grown at $15^{\circ} \mathrm{C}$ until the $\mathrm{L} 4$ stage, and then shifted to $25^{\circ} \mathrm{C}$. For each gene of interest, all RNAi clones available in the two major genome-wide collection $s^{49,50}$ were tested and we then used the ones with the strongest phenotypes. Any RNAi clones that would lead to developmental arrests were retested by initially growing the animals on HT115 bacteria without dsRNAexpressing plasmids at $15^{\circ} \mathrm{C}$ and only at the $\mathrm{L} 4$ stage washing them in antibiotics to remove the HT115 and transferring them to the respective RNAi bacteria and to $25^{\circ} \mathrm{C}$. In all cases, production of progeny was prevented by addition of FUDR $(50$ $\mu \mathrm{M}$ final concentration) at the late L4 stage. GFP expression was evaluated/scored by visual inspection from day 1 to day 3 of adulthood, using a Zeiss Axio Zoom V16 microscope.

mRNA isolation and sequencing. Worms were synchronized by bleaching and about 150 of them grown from the L1 stage at $15^{\circ} \mathrm{C}$ on control, smk-1, or daf- 16 RNAi bacteria. At the L4 stage, the plates were shifted to $25^{\circ} \mathrm{C}$ and FUDR $(50 \mu \mathrm{M}$ final concentration) was added to prevent production of progeny. $16 \mathrm{~h}$ later, 100 animals were collected, washed, and immediately frozen in liquid nitrogen. Total RNA was extracted using Trizol (Sigma). RNA quality was assessed by determining its RNA integrity number (RIN). We required all samples to have a RIN above 9. mRNA-seq libraries were constructed using a TruSeq RNA SamplePrep V2 kit (Illumina) according to the manufacturer's instructions.

Chromatin immunoprecipitation. Approximately 800,000 C. elegans were used per condition in each experiment. Worms were either grown asynchronously at 15 ${ }^{\circ} \mathrm{C}$ on large plates seeded with OP50 bacteria or they were synchronized by bleaching, L1 larvae seeded onto plates containing either control or smk-1 RNA bacteria, and the animals grown at $15^{\circ} \mathrm{C}$ until the late $\mathrm{L} 4$ stage. For the RNA experiments, FUDR ( $50 \mu \mathrm{M}$ final concentration) was added to prevent production of progeny. The animals were then shifted to $25^{\circ} \mathrm{C}$, grown for another $16-18 \mathrm{~h}$, harvested, and subjected to chromatin immunoprecipitation (ChIP) ${ }^{17}$. Briefly, ground frozen worm powder was crosslinked using $1 \%$ formaldehyde in PBS and sonicated. IP was performed using Protein A Dynabeads (Invitrogen) and either rabbit polyclonal anti-GFP antibody (Takara, Cat\# 632592), mouse monoclonal anti-RNA Polymerase II antibody [8WG16] (BioLegend, Cat\# 920102), or mouse monoclonal anti-RNA polymerase II antibody that specifically detects CTD repeats phosphorylated at Ser5 [4H8] (Abcam, Cat\# ab5408). Protein-DNA complexes were then eluted from protein A beads, treated with RNase A and proteinase $\mathrm{K}$, and the resulting DNA purified. Sequencing libraries of inputs and immunoprecipitated samples were constructed using a protocol optimized for small DNA quantities ${ }^{61}$

High-throughput sequencing. For both multiplexed mRNA-seq and ChIP-seq libraries, single-end sequencing was conducted for 50 cycles on Illumina HiSeq 2000 sequencers according to the manufacturer's instructions. Image analysis, base calling, and quality scoring were carried out in real time with the standard Illumina analysis pipeline using a phiX control.

Processing of mRNA-seq reads and downstream analysis. Reads were mapped to the C. elegans genome using TopHat (v2.0.8b) ${ }^{62}$ and known gene model annotations (WS220), as well as the following parameters: --library-type fr-unstranded --b2-very-sensitive --min-coverage-intron 10 --min-segment-intron 10 --microexon-search --no-novel-juncs. Transcript abundance (FPKM, fragments per kilo base of transcript per million fragments) and differential expression was calculated using Cuffdiff (v2.1.1) from the Cufflinks software package ${ }^{63}$ and the following parameters: -u --FDR 0.05 --upper-quartile-norm --compatible-hits-norm --librarytype fr-unstranded. All conditions were analyzed at least by using two biological replicates. Replicates were mapped individually and then combined by Cuffdiff. All analyses were limited to protein-coding genes. Statistically significant differentially expressed genes (DEGs, in other words "up- and downregulated" genes), were identified by Cuffdiff, using a 5\% FDR. Differential gene expression values were calculated as the ratio of FPKM values. To test for significant overlap between gene lists, we used the hypergeometric test ( $\mathrm{R}$ function phyper $^{59}$ ).
Processing of ChIP-seq reads and metagene analysis. For basic quality metrics of our ChIP-seq samples see Supplementary Table 5. We aligned the reads to the $C$. elegans genome (WS220) by using Bowtie (v2.1) ${ }^{64}$ with the following parameter: -q. Uniquely mapping reads that contained no more than one mismatch were used for peak calling and read density calculations. For the calculation of normalized read densities around TSSs, around transcriptional end sites (TESs; the sites where the transcribed region of the mRNA ends and polyadenylation begins), and at DAF-16 peaks, the R package ngs.plot ${ }^{65}$ was used with the following parameters: $-\mathrm{R}$ tss -FL 200 (for TSSs), -R gene body -FL 200 (for TESs), or -R bed -E \{bed-file of DAF16 peaks\} -FL 200 (for DAF-16 peaks). The list of DAF-16 peaks was obtained from ${ }^{17}$. Using this software, read densities were calculated independently for input and IP samples in bins of $60 \mathrm{bp}$ (Fig. 5b-d, Supplementary Figs. 4d and 10a and for the regions outside the gene body in Supplementary Fig. 10b-d) or of $1 \%$ of the gene body length (Supplementary Fig. 10b-d). To then obtain the "fold enrichment over input" values which we plot in the figures, we divide the normalized IP read density of every bin by its corresponding input read density. For $p$ value calculations determining whether DAF-16 binding to DAF-16 peak regions had significantly changed between the compared conditions, we first calculated the "fold enrichment over input" values in $60 \mathrm{bp}$ bins, as stated above, covering a region from -600 to +600 bp around DAF-16 peak summits. Next, we compared the "fold enrichment over input" values in these bins between conditions using a $t$ test $^{66}$. $p$ value calculations determining whether RNA Pol II, RNA Pol II(Ser5Phos), or SPT-5 binding to TSS regions had significantly changed between the compared conditions were conducted in identical manner, only that regions from -600 to $+600 \mathrm{bp}$ around TSSs were investigated.

To calculate pausing indices from RNA Pol II ChIP-seq data, we first created an input-normalized BigWig file of IP data, using DeepTools ${ }^{67}$ (function BamCompare with the following arguments: extendReads 200, operation ratio, binSize 60, scaleFactorsMethod None, normalizeUsing RPKM). For each gene, mean read coverages were calculated in specific regions ("relaxed" pausing region [ -500 to +500 from TSS], "stringent" pausing region [TSS to +500$]$, or the gene body/elongating region [+500 to TES])) using the function mergeByOverlaps from the IRanges package. For each gene, the coverage ratios between the pausing regions and the gene body were calculated independently. Mean ratio values across the gene sets of interest were then used as the index values shown in Supplementary Table 6.

Gene functional enrichment analysis and annotation. Gene functional enrichments were determined using the DAVID Bioinformatics Resources (v. 6.7) ${ }^{68}$. Annotation clusters identified by DAVID (clusters of related annotation terms) having an enrichment score of $\geq 1.3$ were considered significant and a representative naming for the cluster was derived from the contained Gene Ontology terms.

Statistical analysis. Lifespan and stress resistance assays were evaluated by Kaplan-Maier and log-rank tests. Statistical analyses for other experiments are stated in the respective methods sections, tables, or figure legends. Analyses were performed using either SPSS (IBM), OASIS ${ }^{69}$, Excel (Microsoft), Graphpad Prism, or $\mathrm{R}^{59}$.

Reporting summary. Further information on research design is available in the Nature Research Reporting Summary linked to this article.

\section{Data availability}

The high-throughput sequencing data generated and analyzed during this study are available from the authors upon reasonable request as well as from the Sequence Read Archive at NCBI at the following accession code: PRJNA560378. The mass spectrometry data generated and analyzed during this study are available from the authors upon reasonable request as well as from the PeptideAtlas at the following accession code: PASS01428. The source data underlying Figs. 2a-f, 3a-f, 5b, d, 6c-e and Supplementary Figs. $2 a-f, 3 a-c, 4 b-d, 8 b, 9 b, c, e, f$, and 10a are provided as a Source Data file. Noncropped western blot data underlying Supplementary Figs. 7, 8c, and 9a, d are provided in Supplementary Fig. 11 .

Received: 9 December 2018; Accepted: 9 December 2019; Published online: 09 January 2020

\section{References}

1. Kimura, K. D., Tissenbaum, H. A., Liu, Y. \& Ruvkun, G. daf-2, an insulin receptor-like gene that regulates longevity and diapause in Caenorhabditis elegans. Science 277, 942-946 (1997).

2. Tatar, M. et al. A mutant Drosophila insulin receptor homolog that extends life-span and impairs neuroendocrine function. Science 292, 107-110 (2001) 
3. Kenyon, C., Chang, J., Gensch, E., Rudner, A. \& Tabtiang, R. A. C. elegans mutant that lives twice as long as wild type. Nature 366, 461-464 (1993).

4. Lee, S. S., Kennedy, S., Tolonen, A. C. \& Ruvkun, G. DAF-16 target genes that control C. elegans life-span and metabolism. Science 300, 644-647 (2003).

5. Calnan, D. R. \& Brunet, A. The FoxO code. Oncogene 27, 2276-2288 (2008).

6. Murphy, C. T. et al. Genes that act downstream of DAF-16 to influence the lifespan of Caenorhabditis elegans. Nature 424, 277-283 (2003).

7. Lin, K., Hsin, H., Libina, N. \& Kenyon, C. Regulation of the Caenorhabditis elegans longevity protein DAF-16 by insulin/IGF-1 and germline signaling. Nat. Genet. 28, 139-145 (2001).

8. Wolff, S. et al. SMK-1, an essential regulator of DAF-16-mediated longevity. Cell 124, 1039-1053 (2006).

9. Gingras, A.-C. et al. A novel, evolutionarily conserved protein phosphatase complex involved in cisplatin sensitivity. Mol. Cell. Proteom. 4, 1725-1740 (2005).

10. Chowdhury, D. et al. A PP4-phosphatase complex dephosphorylates $\gamma$-H2AX generated during DNA replication. Mol. Cell 31, 33-46 (2008).

11. Chen, G. I. et al. PP4R4/KIAA1622 forms a novel stable cytosolic complex with phosphoprotein phosphatase 4. J. Biol. Chem. 283, 29273-29284 (2008).

12. Janssens, V. \& Goris, J. Protein phosphatase $2 \mathrm{~A}$ : a highly regulated family of serine/threonine phosphatases implicated in cell growth and signalling. Biochem. J. 353, 417-439 (2001).

13. Henderson, S. T. \& Johnson, T. E. daf-16 integrates developmental and environmental inputs to mediate aging in the nematode Caenorhabditis elegans. Curr. Biol. 11, 1975-1980 (2001).

14. Zhou, G. et al. Protein phosphatase 4 is involved in tumor necrosis factoralpha-induced activation of c-Jun N-terminal kinase. J. Biol. Chem. 277, 6391-6398 (2002).

15. Sato-Carlton, A. et al. Protein phosphatase 4 promotes chromosome pairing and synapsis, and contributes to maintaining crossover competence with increasing age. PLoS Genet. 10, e1004638 (2014).

16. Stroustrup, N. et al. The Caenorhabditis elegans Lifespan Machine. Nat. Methods 10, 665-670 (2013).

17. Riedel, C. G. et al. DAF-16 employs the chromatin remodeller SWI/SNF to promote stress resistance and longevity. Nat. Cell Biol. 15, 491-501 (2013).

18. Lin, X.-X. et al. DAF-16/FOXO and HLH-30/TFEB function as combinatorial transcription factors to promote stress resistance and longevity. Nat. Commun. 9, 4400 (2018).

19. Maxwell, C. S. et al. Pol II docking and pausing at growth and stress genes in C. elegans. Cell Rep. 6, 455-466 (2014).

20. Baugh, L. R., Demodena, J. \& Sternberg, P. W. RNA Pol II accumulates at promoters of growth genes during developmental arrest. Science 324, 92-94 (2009).

21. Wade, J. T. \& Struhl, K. The transition from transcriptional initiation to elongation. Curr. Opin. Genet. Dev. 18, 130-136 (2008).

22. Garrido-Lecca, A. \& Blumenthal, T. RNA polymerase II C-terminal domain phosphorylation patterns in caenorhabditis elegans operons, polycistronic gene clusters with only one promoter. Mol. Cell. Biol. 30, 3887-3893 (2010).

23. Chen, R. A. J. et al. The landscape of RNA polymerase II transcription initiation in C. elegans reveals promoter and enhancer architectures. Genome Res. 23, 1339-1347 (2013).

24. Adelman, K. \& Lis, J. T. Promoter-proximal pausing of RNA polymerase II: emerging roles in metazoans. Nat. Rev. Genet. 13, 720-731 (2012).

25. Sacco, F. et al. Combining affinity proteomics and network context to identify new phosphatase substrates and adapters in growth pathways. Front. Genet. 5, 115 (2014).

26. Kaplan, C. D., Morris, J. R., Wu, C. T. \& Winston, F. Spt5 and Spt6 are associated with active transcription and have characteristics of general elongation factors in D. melanogaster. Genes Dev. 14, 2623-2634 (2000).

27. Kato, H. et al. Spt6 prevents transcription-coupled loss of posttranslationally modified histone H3. Sci. Rep. 3, 2186 (2013).

28. Ding, B., LeJeune, D. \& Li, S. The C-terminal repeat domain of Spt5 plays an important role in suppression of Rad26-independent transcription coupled repair. J. Biol. Chem. 285, 5317-5326 (2010).

29. Agostinis, P., Derua, R., Sarno, S., Goris, J. \& Merlevede, W. Specificity of the polycation-stimulated (type-2A) and ATP,Mg-dependent (type-1) protein phosphatases toward substrates phosphorylated by P34cdc2 kinase. Eur. J. Biochem. 205, 241-248 (1992).

30. Zhang, X. et al. Histone deacetylase 3 (HDAC3) activity is regulated by interaction with protein serine/threonine phosphatase 4. Genes Dev. 19 , 827-839 (2005).

31. Lee, D.-H. et al. A PP4 phosphatase complex dephosphorylates RPA2 to facilitate DNA repair via homologous recombination. Nat. Struct. Mol. Biol. 17, 365-372 (2010).

32. Hartzog, G. A. \& Fu, J. The Spt4-Spt5 complex: A multi-faceted regulator of transcription elongation. Biochim. Biophys. Acta 1829, 105-115 (2013).

33. Pavri, R. et al. Activation-induced cytidine deaminase targets DNA at sites of RNA polymerase II stalling by interaction with Spt5. Cell 143, 122-133 (2010).
34. Parua, P. K. et al. A Cdk9-PP1 switch regulates the elongation-termination transition of RNA polymerase II. Nature 558, 460-464 (2018).

35. Fitz, J., Neumann, T. \& Pavri, R. Regulation of RNA polymerase II processivity by Spt5 is restricted to a narrow window during elongation. EMBO J. 37, e97965 (2018).

36. Diamant, G. et al. The elongation factor $\mathrm{Spt} 5$ facilitates transcription initiation for rapid induction of inflammatory-response genes. Nat. Commun. 7, 11547 (2016).

37. Grohmann, D. et al. The initiation factor TFE and the elongation factor Spt $4 / 5$ compete for the RNAP clamp during transcription initiation and elongation. Mol. Cell 43, 263-274 (2011).

38. Yamada, T. et al. P-TEFb-mediated phosphorylation of hSpt5 C-terminal repeats is critical for processive transcription elongation. Mol. Cell 21, 227-237 (2006)

39. Lu, X. et al. Multiple P-TEFbs cooperatively regulate the release of promoterproximally paused RNA polymerase II. Nucleic Acids Res. 44, 6853-6867 (2016).

40. Liu, Y. et al. Phosphorylation of the transcription elongation factor Spt5 by yeast Burl kinase stimulates recruitment of the PAF complex. Mol. Cell. Biol. 29, 4852-4863 (2009).

41. Guo, S. et al. A regulator of transcriptional elongation controls vertebrate neuronal development. Nature 408, 366-369 (2000).

42. Jennings, B. H. et al. Locus-specific requirements for Spt5 in transcriptional activation and repression in Drosophila. Curr. Biol. 14, 1680-1684 (2004).

43. Gaertner, B. \& Zeitlinger, J. RNA polymerase II pausing during development. Development 141, 1179-1183 (2014).

44. Hsu, A.-L., Murphy, C. T. \& Kenyon, C. Regulation of aging and agerelated disease by DAF-16 and heat-shock factor. Science 300, 1142-1145 (2003).

45. Mahat, D. B., Salamanca, H. H., Duarte, F. M., Danko, C. G. \& Lis, J. T. Mammalian heat shock response and mechanisms underlying its genomewide transcriptional regulation. Mol. Cell 62, 63-78 (2016).

46. Ewald, C. Y., Landis, J. N., Porter Abate, J., Murphy, C. T. \& Blackwell, T. K. Dauer-independent insulin/IGF-1-signalling implicates collagen remodelling in longevity. Nature 519, 97-101 (2015)

47. Panowski, S. H., Wolff, S., Aguilaniu, H., Durieux, J. \& Dillin, A. PHA-4/Foxa mediates diet-restriction-induced longevity of C. elegans. Nature 447, 550-555 (2007).

48. Stiernagle, T. Maintenance of C. elegans. WormBook 1, 1-11 (2006).

49. Reboul, J. et al. C. elegans ORFeome version 1.1: experimental verification of the genome annotation and resource for proteomescale protein expression. Nat. Genet. 34, 35-41 (2003).

50. Kamath, R. S. et al. Systematic functional analysis of the Caenorhabditis elegans genome using RNAi. Nature 421, 231-237 (2003).

51. Kennedy, S., Wang, D. \& Ruvkun, G. A conserved siRNA-degrading RNase negatively regulates RNA interference in C. elegans. Nature 427, 645-649 (2004)

52. Hamilton, B. et al. A systematic RNAi screen for longevity genes in C. elegans. Genes Dev. 19, 1544-1555 (2005)

53. Xu, T. et al. ProLuCID, a Fast and Sensitive Tandem Mass Spectra-based Protein Identification Program. Mol. Cell. Proteom. 5, S174 (2006).

54. Tabb, D. L., McDonald, W. H. \& Yates, J. R. DTASelect and Contrast: tools for assembling and comparing protein identifications from shotgun proteomics. $J$ Proteome Res. 1, 21-26 (2002).

55. Frigola, J., Remus, D., Mehanna, A. \& Diffley, J. F. X. ATPase-dependent quality control of DNA replication origin licensing. Nature 495, 339-343 (2013).

56. Kanno, T., Berta, D. G. \& Sjögren, C. The Smc5/6 complex is an ATPdependent intermolecular DNA Linker. Cell Rep. 12, 1471-1482 (2015).

57. Cox, J. \& Mann, M. MaxQuant enables high peptide identification rates, individualized p.p.b.-range mass accuracies and proteome-wide protein quantification. Nat. Biotechnol. 26, 1367-1372 (2008).

58. Cox, J. et al. Andromeda: a peptide search engine integrated into the MaxQuant environment. J. Proteome Res. 10, 1794-1805 (2011).

59. R Core Team. $R$ : a language and environment for statistical computing. http:// www.r-project.org/ (2013).

60. Ritchie, M. E. et al. limma powers differential expression analyses for RNAsequencing and microarray studies. Nucleic Acids Res. 43, e47 (2015).

61. Bowman, S. K. et al. Multiplexed Illumina sequencing libraries from picogram quantities of DNA. BMC Genom. 14, 466 (2013).

62. Trapnell, C., Pachter, L. \& Salzberg, S. L. TopHat: discovering splice junctions with RNA-Seq. Bioinformatics 25, 1105-1111 (2009).

63. Trapnell, C. et al. Transcript assembly and quantification by RNA-Seq reveals unannotated transcripts and isoform switching during cell differentiation. Nat. Biotechnol. 28, 511-515 (2010).

64. Langmead, B., Trapnell, C., Pop, M. \& Salzberg, S. L. Ultrafast and memoryefficient alignment of short DNA sequences to the human genome. Genome Biol. 10, R25 (2009). 
65. Shen, L., Shao, N., Liu, X. \& Nestler, E. ngs.plot: Quick mining and visualization of next-generation sequencing data by integrating genomic databases. BMC Genom. 15, 284 (2014).

66. Whyte, Wa et al. Enhancer decommissioning by LSD1 during embryonic stem cell differentiation. Nature 482, 221-225 (2012).

67. Ramírez, F. et al. deepTools2: a next generation web server for deepsequencing data analysis. Nucleic Acids Res. 44, W160-W165 (2016).

68. Huang, D. W., Sherman, B. T. \& Lempicki, R. A. Systematic and integrative analysis of large gene lists using DAVID bioinformatics resources. Nat. Protoc. 4, 44-57 (2009).

69. Yang, J.-S. et al. OASIS: online application for the survival analysis of lifespan assays performed in aging research. PLoS ONE 6, e23525 (2011).

\section{Acknowledgements}

We thank Proteomics Biomedicum at Karolinska Institute and John Asarah at Harvard Medical School for mass spectrometry analyses. We thank Jianping Liu and Xidan Li at the ICMC sequencing facility, Patrick Muller and Fredrik Fagerström Billai at the Bioinformatics and Expression Analysis Core Facility of Karolinska Institute, and the Lansdorp lab at the European Research Institute for the Biology of Ageing for support with Illumina sequencing. We thank Egon Ogris at the University of Vienna for advising us on in vitro phosphatase assays. We thank Herwig Schüler at Karolinska Institutet for providing TEV protease. We thank the Caenorhabditis Genetic Center (CGC) as well as Shohei Mitani and the National Bioresource Project for C. elegans strains. We thank Mattias Mannervik at Stockholm University for comments on and Christina Jones at Karolinska Institute for language editing of the manuscript. Finally, we thank all Riedel lab members for helpful discussions. Open access funding provided by Karolinska Institute.

\section{Author contributions}

I.S., C.B. and C.G.R. conceived the project and designed the experiments, I.S., X.Z., T.K., A.S., N.P.C. and X.X.L. performed the experiments. A.C. and R.A.Z. conducted the mass spectrometry-based phosphoproteomics analysis. J.S. helped with the analysis of ChIPseq data. B.B. and S.B. provided technical support. I.S. and C.G.R. analyzed the results and wrote the manuscript.

\section{Competing interests}

The authors declare no competing interests.

\section{Additional information}

Supplementary information is available for this paper at https://doi.org/10.1038/s41467019-13931-7.

Correspondence and requests for materials should be addressed to C.G.R.

Peer review information Nature Communications thanks Markku Varjosalo, and the other, anonymous, reviewer(s) for their contribution to the peer review of this work. Peer reviewer reports are available.

Reprints and permission information is available at http://www.nature.com/reprints

Publisher's note Springer Nature remains neutral with regard to jurisdictional claims in published maps and institutional affiliations.

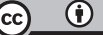

Open Access This article is licensed under a Creative Commons Attribution 4.0 International License, which permits use, sharing, adaptation, distribution and reproduction in any medium or format, as long as you give appropriate credit to the original author(s) and the source, provide a link to the Creative Commons license, and indicate if changes were made. The images or other third party material in this article are included in the article's Creative Commons license, unless indicated otherwise in a credit line to the material. If material is not included in the article's Creative Commons license and your intended use is not permitted by statutory regulation or exceeds the permitted use, you will need to obtain permission directly from the copyright holder. To view a copy of this license, visit http://creativecommons.org/ licenses/by/4.0/.

(C) The Author(s) 2020 